ARTICLE

Received 20 May 2014 | Accepted 17 Dec 2014 | Published 27 Jan 2015

DOI: $10.1038 /$ ncomms7118

\title{
Coexistent ARID1A-PIK3CA mutations promote ovarian clear-cell tumorigenesis through pro-tumorigenic inflammatory cytokine signalling
}

Ronald L. Chandler ${ }^{1,2, \star}$, Jeffrey S. Damrauer ${ }^{1,2, \star}$, Jesse R. Raab ${ }^{1,2}$, Jonathan C. Schisler ${ }^{3,4}$, Matthew D. Wilkerson ${ }^{1,2}$, John P. Didion ${ }^{1,2}$, Joshua Starmer ${ }^{1,2}$, Daniel Serber ${ }^{1,2}$, Della Yee ${ }^{1,2}$, Jessie Xiong ${ }^{2}$, David B. Darr², Fernando Pardo-Manuel de Villena1,2, William Y. Kim,2,5 \& Terry Magnuson ${ }^{1,2}$

Ovarian clear-cell carcinoma (OCCC) is an aggressive form of ovarian cancer with high ARID1A mutation rates. Here we present a mutant mouse model of OCCC. We find that ARIDIA inactivation is not sufficient for tumour formation, but requires concurrent activation of the phosphoinositide 3-kinase catalytic subunit, PIK3CA. Remarkably, the mice develop highly penetrant tumours with OCCC-like histopathology, culminating in haemorrhagic ascites and a median survival period of 7.5 weeks. Therapeutic treatment with the pan-PI3K inhibitor, BKM120, prolongs mouse survival by inhibiting the tumour cell growth. Crossspecies gene expression comparisons support a role for IL-6 inflammatory cytokine signalling in OCCC pathogenesis. We further show that ARID1A and PIK3CA mutations cooperate to promote tumour growth through sustained IL-6 overproduction. Our findings establish an epistatic relationship between SWI/SNF chromatin remodelling and PI3K pathway mutations in OCCC and demonstrate that these pathways converge on pro-tumorigenic cytokine signalling. We propose that ARID1A protects against inflammation-driven tumorigenesis.

\footnotetext{
${ }^{1}$ Department of Genetics, University of North Carolina at Chapel Hill, Chapel Hill, North Carolina 27599, USA. ${ }^{2}$ Lineberger Comprehensive Cancer Center, University of North Carolina at Chapel Hill, Chapel Hill, North Carolina 27599, USA. ${ }^{3}$ McAllister Heart Institute, University of North Carolina at Chapel Hill, Chapel Hill, North Carolina 27599, USA. ${ }^{4}$ Department of Pharmacology, University of North Carolina at Chapel Hill, Chapel Hill, North Carolina 27599 , USA. ${ }^{5}$ Department of Medicine, University of North Carolina at Chapel Hill, Chapel Hill, North Carolina 27599, USA. * These authors contributed equally to this work. Correspondence and requests for materials should be addressed to T.M. (email: trm4@med.unc.edu).
} 
pithelial ovarian cancer (EOC) ranks as the fifth leading - cause of cancer death among women ${ }^{1}$. EOC consists of four major histologic tumour subtypes (serous, clear-cell, endometrioid or mucinous) and originates from the coelomic epithelium, which is the precursor to the ovarian surface epithelium (OSE) and Müllerian ductal epithelium ${ }^{2}$. The salpinge or fallopian tube of the Müllerian epithelial anlage is the more definitive source of serous $\mathrm{EOC}^{3}$. Endometriotic explants originating from more distal sites in the Müllerian epithelial anlage may have some propensity to give rise to clear-cell or endometrioid EOC in humans, but this alternate mechanism of pathogenesis has not been thoroughly investigated $^{4,5}$.

In addition to the histologic feature, each EOC subtype can be further classified into two distinct groups based on the disease progression, metastatic potential and molecular signature, with low-grade serous, clear-cell, mucinous and endometrioid subtypes being classified as Type I tumours and the high-grade serous subtype as Type II tumours ${ }^{6}$. In general, Type I tumours account for up to $25 \%$ of EOCs, are genomically stable and have lower metastatic potential than Type II tumours, which constitute most of the remaining EOC cases $^{6}$. In addition, KRAS, BRAF, PIK3CA, PTEN and CTNNB1 ( $\beta$-CATENIN) mutations have historically been associated with Type I tumours at varying frequencies, whereas Type II tumours are largely defined by high-frequency TRP53 (p53) mutations and chromosome instability ${ }^{6-8}$. Therefore, EOC consists of a heterogeneous group of cancers, each with unique clinical challenges and biology.
OCCC is the most common Type I tumour, accounting for $5-25 \%$ of all EOC cases, with incidence varying between populations ${ }^{9-11}$. Endometriosis is recognized as a significant risk factor for OCCC ${ }^{12-14}$. Among all EOC subtypes, OCCC has the worst prognosis if diagnosed at an advanced stage of disease because of poor response rates to platinum-based chemotherapy $9,10,15,16$. Consequently, survival rates for women with advanced stage OCCC are low ${ }^{9,10,15,16}$. Although OCCC is the second leading cause of death from ovarian cancer, the aetiology and pathogenesis of this devastating disease are poorly understood.

Recent genome-sequencing efforts support a strong genetic contribution to OCCC aetiology based on the discovery of high-frequency (up to 50\%) ARID1A tumour mutations ${ }^{17,18}$. ARID1A is also somatically mutated in another Type I EOC, endometrioid carcinoma and several other gynaecologic cancers, including uterine clear-cell and endometrioid carcinomas, cervical carcinoma and uterine carcinosarcoma ${ }^{19,20}$. Additional tumour-sequencing studies have detected recurrent ARID1A mutations in several non-gynaecologic cancers and, taken together, components of the SWI/SNF complex are mutated in at least $20 \%$ of human cancers ${ }^{19,21-23}$. ARID1A is a subunit within the SWI/SNF chromatin remodelling complex that facilitates target substrate recognition; however, its role in OCCC tumour initiation and progression has, yet, to be fully elucidated ${ }^{24}$.

Genetically engineered mouse models offer the opportunity to investigate the contribution of genetic factors to EOC aetiology a

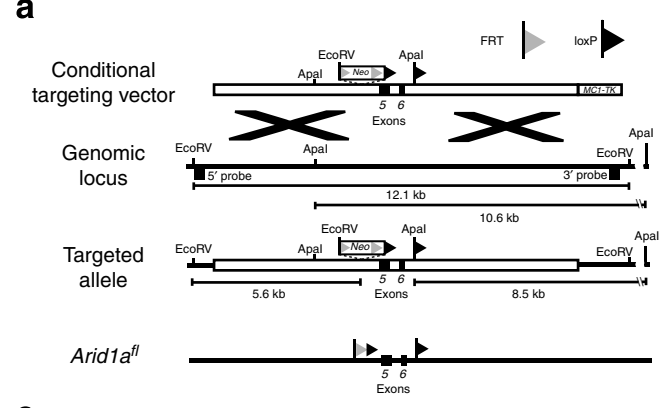

C

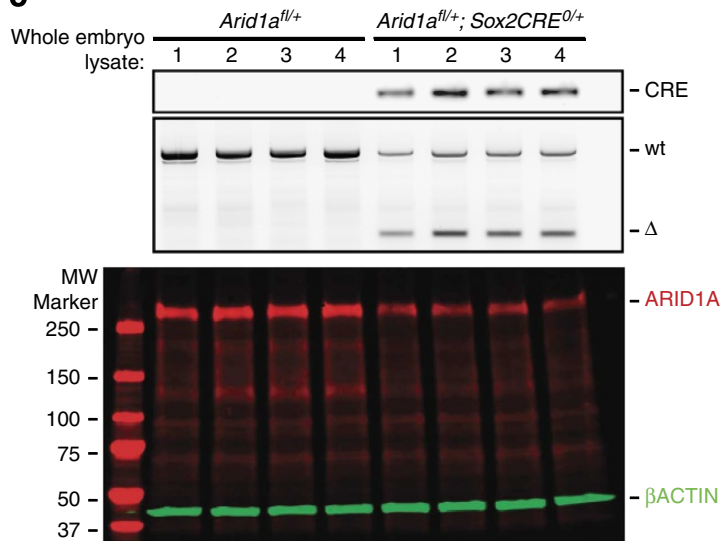

b

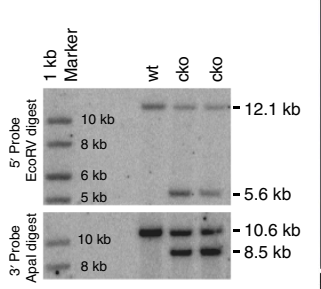

d

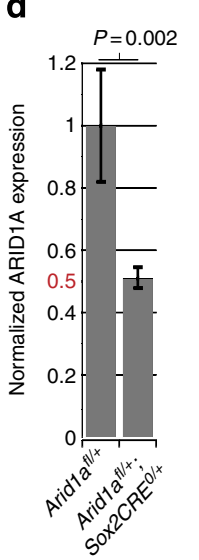

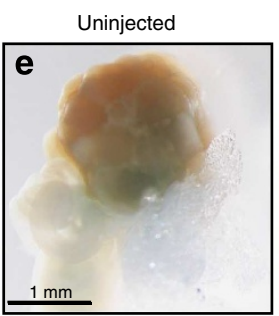

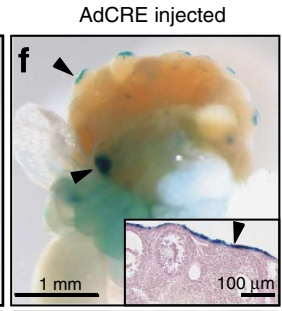

g

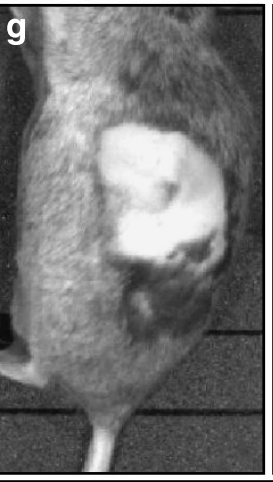

h

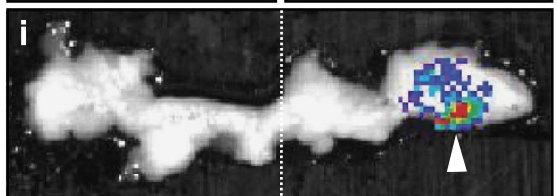

Figure 1 | A new Arid1a conditional allele to explore ARID1A tumour-suppressor function in vivo. (a) Targeting scheme to insert two loxP sites flanking exon 5 and 6 of mouse ARID1A. (b) Representative Southern blot showing properly targeted embryonic stem cell clones. Insertion of loxP sites generates $5.6 \mathrm{~kb}$ EcoRV and $8.5 \mathrm{~kb}$ Apal genomic fragments. (c) PCR genotyping and quantitative, dual-colour fluorescent western blot of ARID1A (red) and BACTIN (green) protein expression in whole-embryo lysates from four Arid1a ${ }^{f l /}+$ and four Arid1a ${ }^{f l /+} ;$ Sox $2 \mathrm{Cre}{ }^{0 /+}$ embryos. (d) Graph of quantitative western blot results depicting normalized protein levels. Significant differences based on the average normalized protein expression \pm s.d. of four independent embryos were calculated using a two-tailed Student's $t$-test ( $P$ values $<0.05$ were considered significant). (e,f) Representative LacZ-stained, control (uninjected) or intrabursal AdCRE-injected (Gt)Rosa26lacZ ovary showing patterns of CRE-mediated recombination in the OSE. (f, inset) Histological section of LacZ-stained AdCRE-injected (Gt)Rosa26lacZ ovary. (g-i) Live-luminescence overlaid whole-mount images of an intrabursal AdCRE-injected, (Gt)Rosa26luciferase female mouse. (i) Excised reproductive tract. Asterisks in $\mathbf{h}$ indicate wound staple. 
and pathogenesis ${ }^{25}$. In this regard, early research using mouse models of EOC established causative roles for coexistent mutations in PTEN and KRAS or PTEN and WNT/ $\beta$ CATENIN pathways in endometrioid $\mathrm{EOC}^{7,8}$. Recently, Guan et $a .^{26}$ reported a mouse model of endometrioid EOC involving coexistent mutations in PTEN and ARID1A. In this study, we describe a new mouse model that genetically and histologically resembles human OCCC and functionally implicates coexistent ARID1A and PIK3CA mutations in cancer causation. We further provide new mechanistic insight into OCCC pathogenesis by establishing a functional link between coexistent ARID1APIK3CA mutations and interleukin-6 (IL-6) signalling.

\section{Results}

Generation of a novel Arid1a conditional allele. To establish a functional role for ARID1A mutations in ovarian clear-cell tumorigenesis, we generated a novel Arid1a conditional allele (Aridla $a^{f l}$ ) by inserting loxP sites flanking exons 5 and 6 in ARID1A (Fig. 1a,b). Arid1a-null embryos die around gastrulation and thus they are not amenable to efficient protein extraction ${ }^{27}$. In light of its early embryonic lethality, we monoalleliclly inactivated ARID1A in embryonic tissues (via Sox2Cre ${ }^{28}$ ) to verify our Arid1afl allele in the whole animal. Using this approach, we observed an expected 50\% reduction in total protein from whole-embryo lysates (Fig. 1c,d). We next sought to inactivate ARID1A in the OSE. To do this, we employed the intrabursal AdCRE recombination system ${ }^{7,29}$. Efficient CREmediated genetic recombination was consistently observed in the OSE and outer epithelial layers of tissues confined to the bursal space (Fig. 1f,h,i). We performed a series of intrabursal AdCRE injections on two independent cohorts of Arid1 $a^{f l f l}$ mice $(n=42)$ and followed them for $\sim 1$ year. The mice remained tumour free with no significant changes in survival, other than age-related mortality, similar to the phenotypes reported by Guan et al. ${ }^{26}$ (Supplementary Fig. 1). The observation that ARID1A loss does not lead to tumour formation supports the notion that ARIDIA mutations require additional mutational 'hits' in the OSE before tumorigenesis can ensue ${ }^{26}$.

Coexistent ARID1A-PIK3CA mutations initiate ovarian cancer. To explore this further, we focused on the cancer proto-oncogene, PIK3CA (Phosphatidylinositol-4,5-bisphosphate 3-kinase, catalytic subunit alpha or $\mathrm{p} 110 \alpha$ ), which also has high OCCC mutation rates ${ }^{30,31}$. Recently, coexistent ARID1A-PIK3CA mutations were reported in gastric cancer ${ }^{32}$. In addition, it has been observed that OCCC tumours harbouring PIK3CA mutations display a concomitant loss of ARID1A immunoreactivity, raising the possibility that ARID1A and PIK3CA mutations frequently co-occur in OCCC ${ }^{33}$. To understand the degree of mutational co-occurrence, we compared the incidence of ARID1A and/or PIK3CA mutations in available tumour data sets curated in The Cancer Genome Atlas (TCGA) to OCCC tumour-sequencing data ${ }^{17}$. We found that the majority of cancers carry genetic alterations in one of these genes; however, the rate of ARID1A-PIK3CA mutational co-occurrence $(33 \% ; P<0.05)$ is highest in OCCC (Fig. 2a).

PIK3CA tumour mutations often result in an H1047R 'hotspot' substitution within the kinase domain, generating a catalytically active protein ${ }^{34}$. PIK3CA amplification also frequently occurs in $\mathrm{EOC}^{30}$. To investigate the effect of PIK3CA alterations on ovarian tumorigenesis, we used the CRE-inducible (Gt)Rosa26Pik3ca ${ }^{* H 1047 R}$ allele ${ }^{35}$ to drive PIK3CA ${ }^{\text {H1047R }}$ expression from the Rosa26 locus. We observed multifocal sites of epithelial hyperplasia in the OSE of AdCRE-injected (Gt)Rosa26Pik3ca ${ }^{+H 1047 R}$ mice, but no tumour formation
(Table 1). Intrabursal AdCRE induction of PIK3CA ${ }^{\mathrm{H} 1047 \mathrm{R}}$, under the control of the endogenous promoter, also led to OSE hyperplasia in the mouse $\mathrm{e}^{36}$, suggesting that constitutive overexpression of PIK3CA ${ }^{\text {H1047R }}$ from the Rosa26 locus does not further augment this process.

We next evaluated the combinatorial effects of ARID1A loss and PIK3CA activation by performing AdCRE injections into the ovarian bursa of Aridla ${ }^{f l / f l}$; $(G t) R o s a 26 P i k 3 c a^{*} H 1047 R$ mice. In stark contrast to the single mutants, Arid1 fllfl $^{\text {f }}(\mathrm{Gt})$ Rosa26Pik3$c a^{* H 1047 R}$ mice rapidly developed primary ovarian tumours and showed evidence of abdominal distension, warranting killing with a median latency period of 7.5 weeks post AdCRE injection (Fig. 2j-m). Evidence for tumour-specific ARID1A loss and PIK3CA ${ }^{\mathrm{H} 1047 \mathrm{R}}$ expression or PI3K pathway activation was detected by PCR amplification of tumour complementary DNA (cDNA) or by tumour immunohistochemistry (IHC) for ARID1A and phosphorylation of AKT at serine 473 (P-AKT S473; Fig. 2b-i). Increased morbidity coincided with the presentation of haemorrhagic ascites (Fig. 2j,l). Peritoneal metastases were detected in $\sim 50 \%$ of the double mutants, often residing near the contralateral, uninjected ovary (Fig. $2 \mathrm{k}$, see Table 1). Distant metastases beyond the abdominal cavity were not evident on gross examination. We did not find evidence for genome instability in Arid1 $a^{f l / f l}$; (Gt)Rosa26Pik3ca ${ }^{* H 1047 R}$ tumour samples using mouse high-density SNP (single-nucleotide polymorphism) arrays (Supplementary Fig. 2).

ARID1A haploinsufficiency does not lead to tumour formation. Several studies point to a gene dose-dependent role for ARID1A in tumour suppression given the high incidence of heterozygous tumour mutations in cancer ${ }^{19}$. To determine if an ARID1A haploinsufficiency coupled with PI3K pathway activation leads to ovarian cancer in our model, we performed AdCRE injections on Arid1a ${ }^{f l+} ;(G t) R o s a 26 P i k 3 c a^{* H 1047 R}$ mice. OSE hyperplasia was observed in five out of the seven Aridla ${ }^{f l}+$; (Gt)Rosa26Pik3ca ${ }^{* H 1047 R}$ mice, but evidence of tumour formation was not detected after the 11-week observation period, similar to the (Gt)Rosa26Pik3ca ${ }^{*} H 1047 R$ only cohort (Fig. 2n,o, see Table 1). These data are consistent with the observation that most OCCC tumours carrying heterozygous mutations in ARID1A show the loss of ARID1A immunoreactivity and support the notion that additional mechanisms lead to the loss of ARID1A protein expression in OCCC $^{18,37}$. Thus, ARID1A likely follows the classical 'two-hit' model of tumour suppression in OCCC.

Therapeutic PIK3CA inhibition prolongs animal survival. We next sought to examine the role of continued PIK3CA activation in the progression of ARID1A-deficient ovarian tumours and determine whether therapeutic PIK3CA inhibition would inhibit tumour cell growth and improve animal survival. Primary EOC tumour cells were isolated from the exfoliated tumour cell aggregates present in ascites fluid of tumour-burdened Arid1 $1 a^{f l f l}$; (Gt)Rosa26Pik3ca ${ }^{*}$ 1047R mice and subcultured using established methods (Fig. 3a). Isolated tumour cells displayed ARID1A loss and heightened PI3K signalling, as evidence by the loss of ARID1A immunoreactivity and increased P-AKT S473 levels compared with normal OSE cells (Fig. 3b). Administration of the pan-class I PI3K inhibitor, BKM120 (Buparlisib) led to a dosedependent decrease in P-AKT S473 and concomitant reduction in tumour cell viability $\left(\mathrm{IC}_{50}=0.96 \pm 0.25 \mu \mathrm{M}\right.$; Fig. 3c,d). We next administered chow-fed BKM120 to Arid1a fl/fl; (Gt)Rosa26Pik $3 \mathrm{ca}^{{ }^{*} H 1047 R}$ mice for 3 weeks, starting at week 4 post AdCRE injection. The effectiveness of BKM120-dependent inhibition of PI3K signalling in chow-fed mice was accessed by tumour IHC 

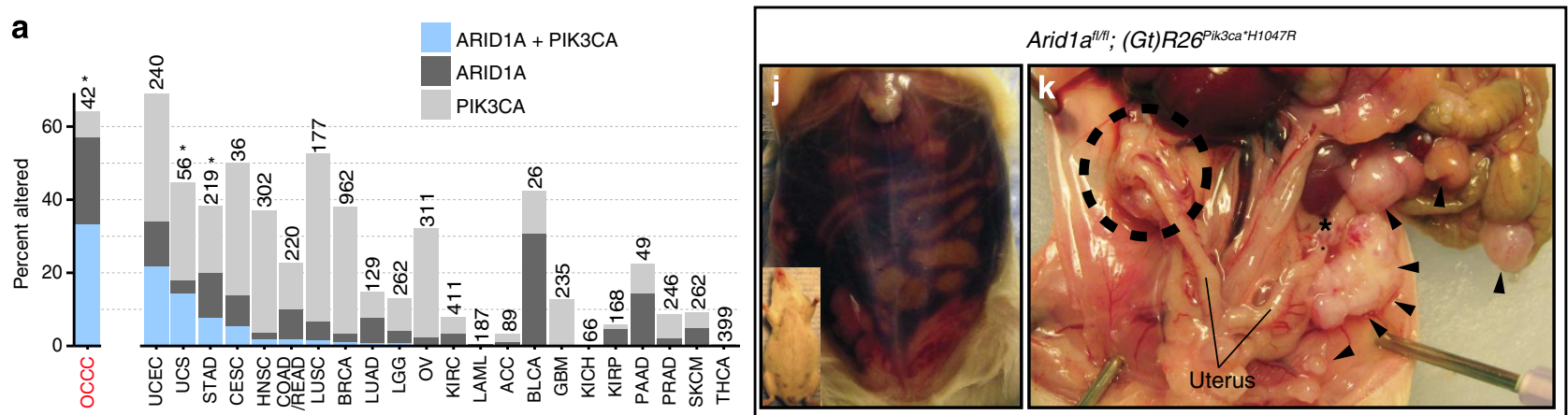

b
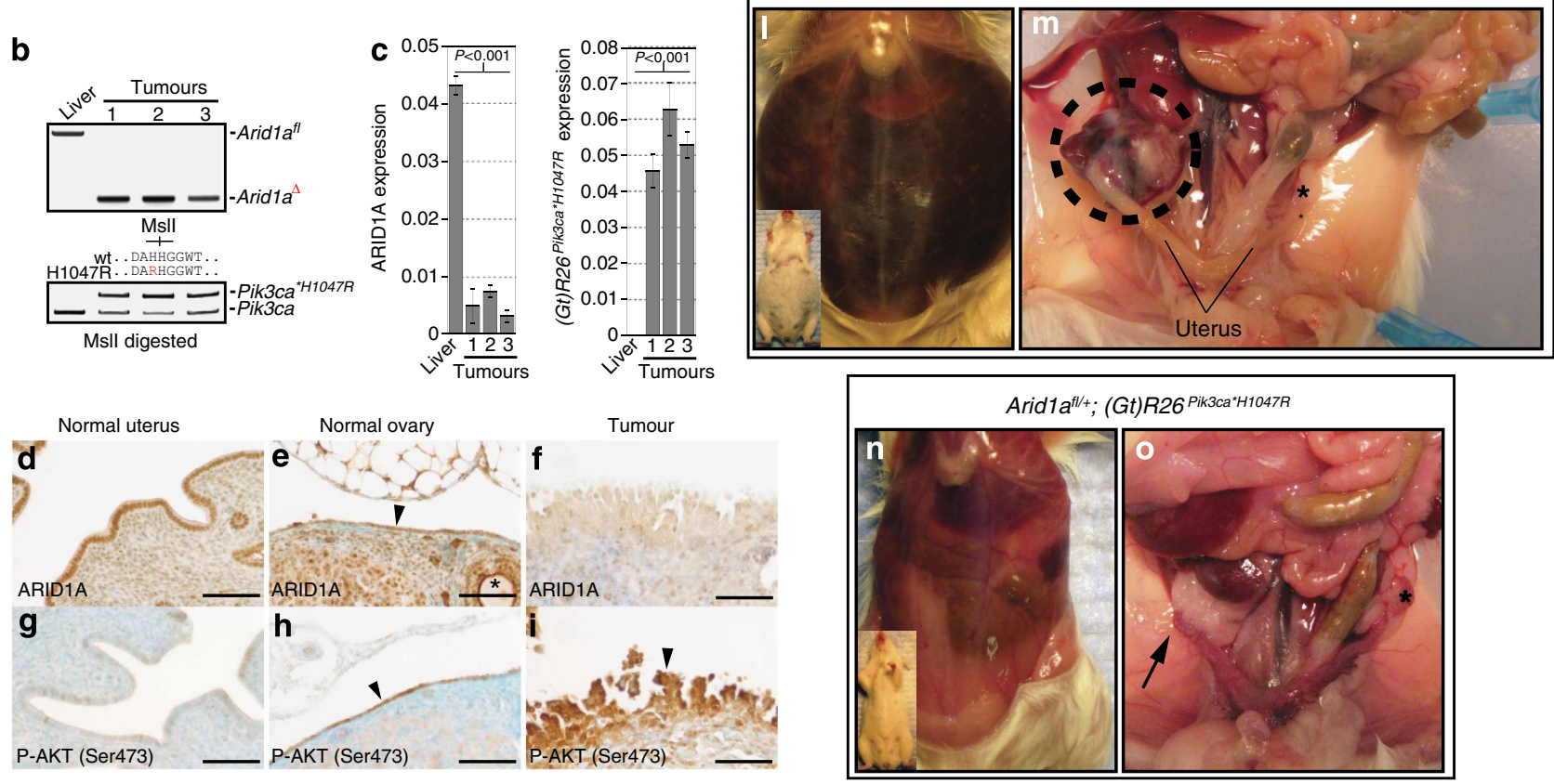

Figure 2 | Concurrent ARID1A loss and PIK3CA activation leads to ovarian tumorigenesis in the mouse. (a) Plot of ARID1A and PIK3CA alterations across available TCGA data sets. Mutation or co-mutation frequencies are expressed as a percentage of all tumour samples for each cancer. A Fisher's exact test was used to calculate the significance of association between ARID1A and PIK3CA mutations ( ${ }^{\star} P$ value $<0.05$ ). (b) Genotyping scheme to detect CRE-deleted (Arid1a4) allele in tumour genomic DNA samples. RT-PCR was used to distinguish PIK3CA and transgenic (Gt)Rosa26Pik3ca ${ }^{*}+1047 R$ transcripts in tumour RNA samples. The H1047R mutation in (Gt)Rosa26Pik3ca ${ }^{*}$ H1047R protects against Msll digest of amplified tumour cDNA. (c) ARID1A loss or (Gt)Rosa26Pik3ca ${ }^{\star}$ H1047R transcripts were detected in tumour RNA samples by RT-PCR. Significant differences based on the average normalized mRNA expression \pm s.d. between replicates of a control liver sample and replicates of three independent tumour samples were calculated using a twotailed Student's $t$-test ( $P$ values $<0.05$ were considered significant). (d,e) ARID1A expression is observed in cells throughout the normal uterus and ovary by IHC. (e) ARID1A is expressed in the OSE of normal ovaries (arrowhead). (f) ARID1A expression is not observed in the tumours. (g) P-AKT S473 levels are low in the normal uterus. (h,i) P-AKT S473 in the normal ovary is highest in the OSE (h, arrowhead) and these levels are greatly increased in ovarian tumours (i, arrowhead). Asterisk in e denotes an oocyte. All sections processed for IHC were lightly counterstained with methyl green. (j,k) Morbid Arid1a ${ }^{f l / f l} ;(G t) R o s a 26 P i k 3 c a^{*} H 1047 R$ mouse at killing with haemorrhagic ascites (inset), primary ovarian tumour of moderate size and bilateral tumour metastases (arrowheads). (I, m) Morbid Arid1a $a^{f l / f l} ;(G t) R o s a 26 P i k 3 c a^{\star H 1047 R}$ mouse at killing with haemorrhagic ascites (inset), large primary ovarian tumour and no visible metastases. The mice shown in $\mathbf{j}-\mathbf{m}$ were killed at 7 and 9 weeks post AdCRE, respectively, because of visible ascitic fluid burden. $(\mathbf{n}, \mathbf{0})$ Arid1 $a^{f l}+;$;Gt)Rosa26Pik3ca ${ }^{\star H 1047 R}$ mice at 11 weeks post AdCRE showing no evidence for tumour formation. In $\mathbf{k}, \mathbf{m}$, dashed circles indicate primary ovarian tumour on injected ovary. In $\mathbf{n}$, arrows denote the AdCRE-injected ovary. In $\mathbf{k}, \mathbf{m}, \mathbf{o}$, asterisks denote the uninjected, control ovary. ACC, adrenocortical carcinoma; BLCA, bladder urothelial carcinoma; BRCA, breast invasive carcinoma; CESC, cervical squamous cell carcinoma and endocervical adenocarcinoma; COAD/READ, colon and rectum adenocarcinoma; GBM, glioblastoma multiforme; HNSC, head and neck squamous cell carcinoma; $\mathrm{KICH}$, kidney chromophobe; KIRC, kidney renal clear-cell carcinoma; KIRP, kidney renal papillary cell carcinoma; LAML, acute myeloid leukaemia; LGG, brain lower-grade glioma; LUAD, lung adenocarcinoma; LUSC, lung squamous cell carcinoma; OV, ovarian serous cystadenocarcinoma; PAAD, pancreatic adenocarcinoma; PRAD, prostate adenocarcinoma; SKCM, skin cutaneous melanoma; STAD, stomach adenocarcinoma; THCA, thyroid carcinoma; UCEC, uterine corpus endometrial carcinoma; UCS, uterine carcinosarcoma.

for phosphorylation of the S6 ribosomal protein at serine 235/236 (P-S6; Fig. 3e). BKM120 treatment led to reduced P-S6 levels and extended the median latency period by 3.5 weeks (Fig. 3e,f). These data indicate that therapeutic PI $3 \mathrm{~K}$ inhibition promotes animal survival by inhibiting tumour cell growth, thus providing strong rationale for the use of PI3K inhibitors in OCCC treatment.
Mouse ovarian tumours manifest OCCC-like histopathology. Cardinal histopathological features of human OCCC include cells with clear cytoplasm, stromal hyalinization, solid, papillary or tubulocystic tumour architectural patterns, and HNF1 $\beta$ immunoreactivity $^{38}$. The ovarian tumours observed in Aridla fl/fl; (Gt)Rosa26Pik3ca ${ }^{\star} H 1047 R$ mice were predominately solid in 
Table 1 | Ovarian tumorigenesis requires concurrent ARID1A loss and PIK3CA activation.

\begin{tabular}{|c|c|c|c|c|c|}
\hline & $\begin{array}{c}{ }^{\star}{ }_{\text {Arid1afl/fl, }} \text {; } \\
\text { Gt(Rosa)26 }{ }^{\text {Pik3ca }}{ }^{\star} H 1047 R\end{array}$ & $\begin{array}{c}{ }^{\star} \text { BKM120-treated Arid1a }{ }^{f l / f l} ; \\
\text { Gt(Rosa)26 }\end{array}$ & $\begin{array}{c}\text { Arid1a fl }+; \\
\text { Gt(Rosa)26 } \\
\text { Pik3ca*H1047R }\end{array}$ & ${ }^{\dagger}$ Arid1a ${ }^{f l} / f l$ & ${ }^{\dagger} \mathrm{Gt}($ Rosa $) 26^{\text {Pik3ca }{ }^{\star} H 1047 R}$ \\
\hline OSE hyperplasia ${ }^{\ddagger}$ & - & - & $5 / 7(71 \%)$ & $0 / 6(0 \%)$ & $4 / 5(80 \%)$ \\
\hline Ascites & $12 / 30 \S(40 \%)$ & $6 / 10^{\| 1}(60 \%)$ & $0 / 8(0 \%)$ & $0 / 47^{\top}(0 \%)$ & $0 / 6(0 \%)$ \\
\hline $\begin{array}{l}\text { Primary ovarian } \\
\text { tumours (injected side) }\end{array}$ & $23 / 30^{\#,{ }^{\star \star}, \dagger \dagger}(77 \%)$ & $8 / 10^{\| 1}(80 \%)$ & $0 / 8(0 \%)$ & $0 / 47^{\top}(0 \%)$ & $0 / 6(0 \%)$ \\
\hline Papillary/solid $\ddagger$ & $6 / 12(50 \%)$ & - & - & - & - \\
\hline Tubulocystic ${ }^{\ddagger}$ & $2 / 12(17 \%)$ & - & - & - & - \\
\hline Mixed $\ddagger$ & $4 / 12(33 \%)$ & - & - & - & - \\
\hline Peritoneal metastases & $17 / 30^{\#,{ }^{\star \star}, \dagger \dagger}(57 \%)$ & $5 / 8(63 \%)$ & $0 / 8(0 \%)$ & $0 / 47^{\top}(0 \%)$ & $0 / 6(0 \%)$ \\
\hline $\begin{array}{l}\text { Contralateral ovarian } \\
\text { metastases }\end{array}$ & $4 / 30^{\#,{ }^{* \star}, \dagger}(13 \%)$ & $0 / 8(0 \%)$ & $0 / 8(0 \%)$ & $0 / 47^{\top}(0 \%)$ & $0 / 6(0 \%)$ \\
\hline $\begin{array}{l}\text { Contralateral peritoneal } \\
\text { metastases }\end{array}$ & $15 / 30^{\#,{ }^{\star \star},+\dagger}(50 \%)$ & $4 / 8(50 \%)$ & $0 / 8(0 \%)$ & $0 / 47^{\top}(0 \%)$ & $0 / 6(0 \%)$ \\
\hline \multicolumn{6}{|c|}{$\begin{array}{l}\text { OSE, ovarian surface epithelium. } \\
\star \text { Only morbid-euthanized mice were scored. } \\
\text { †Mice were euthanized and scored at the end of the 11-week observation period. } \\
\text { †Scored by histology. } \\
\text { \$Three mice found dead and not scored for ascites. } \\
\text { \|Two mice found dead after treatment and not scored for ascites or primary tumours. } \\
\text { TMice aged } 1 \text { year included. } \\
\text { \#Four mice were found dead and not scored for tumour histology. } \\
\star \star * \text { Two mice had tumours within the body wall at incision site that were likely the result of leaky AdCRE and were not scored. } \\
\dagger \text { One mouse had a very large uterine tumour that was likely a result of leaky AdCRE into the lumen of the oviduct or uterus and was not scored. }\end{array}$} \\
\hline
\end{tabular}

appearance with some papillary areas (Fig. 4a-d, see Table 1). Tubulocystic patterns were rarely observed (Fig. 4e, see Table 1). Neoplastic cells with clear cytoplasm, prominent nucleoli and pleomorphic nuclei were observed in the tumours (see high magnification in Fig. $\left.4 a^{\prime}-c^{\prime}\right)$. Elongated spindle-shaped cells with finely vacuolated cytoplasm were observed embedded in hyalinized matrix. Solid clear-cell carcinomas were observed directly attached to the ovarian surface (Fig. 4a). We rarely observed tumour cells organized into bands of glandular epithelium with distinct borders, and, in general, the tumours appeared poorly differentiated and highly disorganized. Primary tumour masses ranged from $\sim 100 \mathrm{mg}$ to over $1 \mathrm{~g}$ (Fig. $4 \mathrm{j}$ ). Differences in tumour size, metastatic potential or morbidity did not correlate with any particular histologic feature.

In human OCCC, neoplastic cells that line the luminal spaces often assume a 'hobnail' appearance, which crudely resembles a nucleus standing on the tip of a cytoplasmic stalk. Hobnail cells were often observed on the tumour periphery in regions where tumour cell exfoliation into luminal regions was occurring (Fig. 4d,i,k). Exfoliated, metastatic tumour cell aggregates often consisted of hobnail-shaped cells organized around either clearcell or hyalinized cores (Fig. 4i). OSE hyperplasia and small clumps of hobnail cells were evident on the ovarian surface as early as 1 week following AdCRE injection, with exfoliated tumour cell aggregates and clear-cell-like features apparent at 2 weeks (Supplementary Fig. 3). The tumours were also positive for the human OCCC marker, HNF1 $\beta$, by IHC (Fig. 5b). Thus, mouse ovarian tumours harbouring coexistent ARID1A-PIK3CA mutations share histopathological features with human OCCC.

Tumour gene expression supports a role for IL-6 in OCCC. To further evaluate the relevance of our Arid1a $a^{f l f l} ;(G t)$ Rosa26$P i k 3 \mathrm{ca}^{*}{ }^{*} 1047 R$ ovarian tumour model to human OCCC, we performed a series of cross-species gene expression comparisons using a well-characterized gene expression data set containing all four human EOC subtypes ${ }^{39}$. Gene expression profiles of mouse primary tumour samples and matched normal (uninjected) ovaries were generated by microarray. Consistent with our genetic model, ARID1A and PIK3CA expression levels were inversely correlated in comparisons between tumour and normal ovary samples, with PIK3CA upregulation strongly correlated with ARID1A downregulation in the tumour samples (Supplementary Fig. 4). We verified our microarray gene expression results by reverse transcription-PCR (RT-PCR) validation of KRAS, TFPI2, CDKN2A (P16), CDKN1A (P21), MUC16 (CA125) and VCAN expression (Supplementary Fig. 5). We next identified all coordinately regulated genes in both mouse tumours and human OCCC, as compared with species-matched normal ovaries. In comparisons of both mouse and human data sets, the control ovary samples showed high levels of gene expression variation, suggesting tissue sampling differences or general interspecies-specific gene expression differences (Supplementary Fig. 6). Focusing on those genes that remained unchanged in normal mouse and human ovaries, we identified 584 coordinately regulated genes (272 upregulated; 312 downregulated) in mouse and human tumours (Supplementary Fig. 7). Mouse and human tumour gene expression patterns were enriched for genes involved in IL-6 signalling, immune system function, focal adhesion and regulation of the actin cytoskeleton, as compared with normal ovaries (Supplementary Figs 6 and 7).

Since many of the upregulated genes we identified in crossspecies comparisons with normal ovaries are likely to be found in other EOC subtypes (for example, CA125) or generally found across most cancers, we next sought to identify an OCCC-specific gene signature for use in subtype-specific gene expression comparisons with the mouse tumours. To do this, we compiled a more refined 'discriminant' gene list for each subtype (for example, clear-cell versus all other EOC subtypes). We then compared the normalized raw expression values for each discriminant gene across all of the mouse tumour and human EOC samples. Using this analysis, we found that the gene expression patterns for mouse tumour and human OCCC samples were not statistically different $(P=0.6)$, suggesting that the OCCC-specific gene expression patterns are conserved between the two species (Fig. 5c). Several well-known markers of OCCC were identified as being highly expressed in the mouse tumours, including IL-6, STAT3, VCAN and HIF1 $\alpha$, further supporting a role for heightened IL-6 signalling in OCCC tumour pathogenesis $^{40-42}$ (Fig. 6a). 

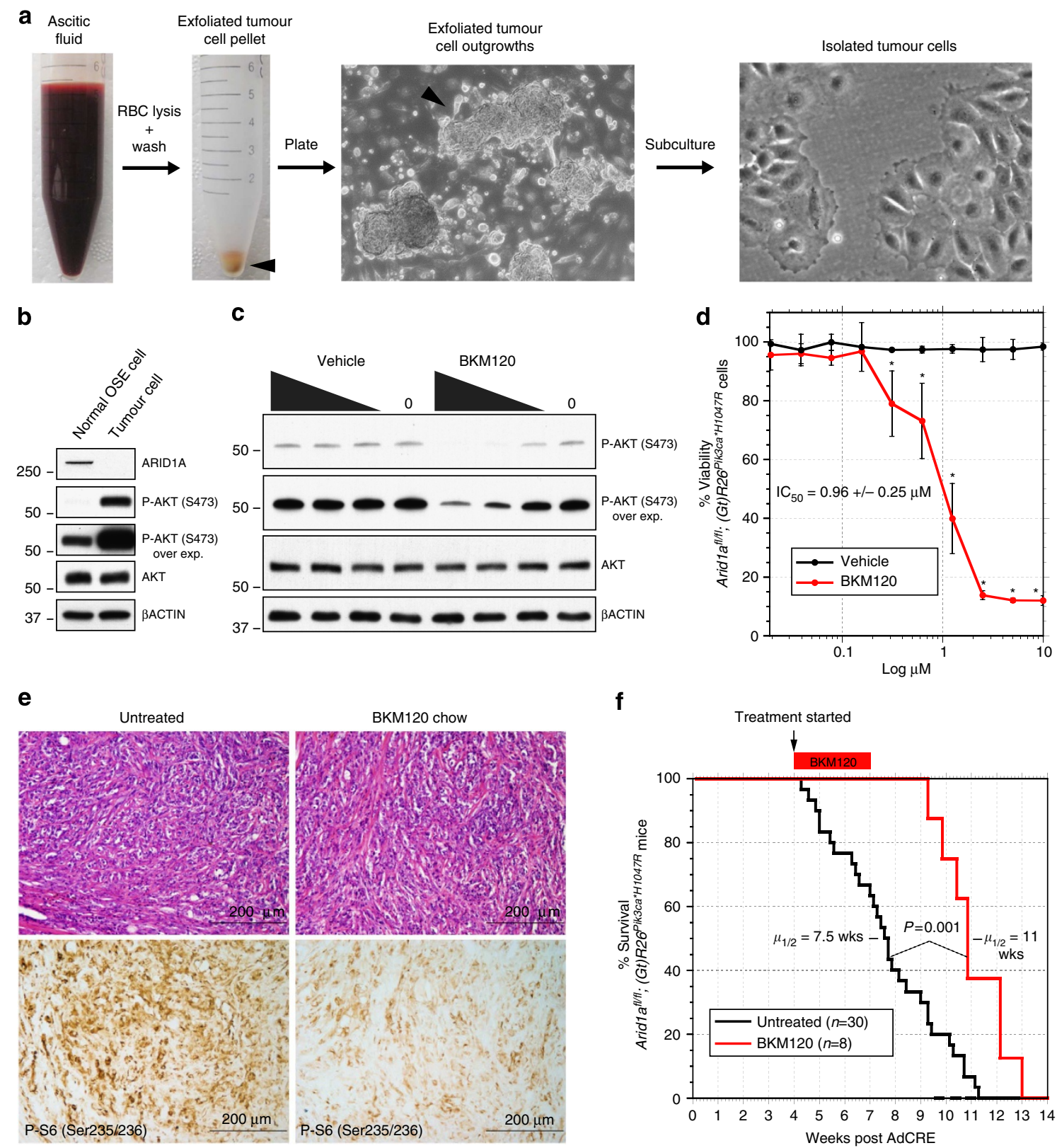

Figure 3 | Therapeutic PIK3CA inhibition promotes animal survival by reducing tumour cell viability. (a) Primary ascites-derived tumour cell isolation scheme. (b) Representative western blots to highlight the primary tumour cell validation scheme and demonstrate that ARID1A loss and heightened P-AKT S473 is observed in primary Arid1a ${ }^{\mathrm{fl} / \mathrm{fl} ;}$ (Gt)Rosa26Pik3ca ${ }^{*} H 1047 R$ ascitic tumour cells versus normal OSE cells. (c) Western blots of P-AKT S473 and total AKT levels in Arid1a ${ }^{f l / f l}$; (Gt)Rosa26Pik3ca ${ }^{*} H 1047 R$ ascitic tumour cells grown in the presence 10, 1, 0.001 or $0 \mu \mathrm{M}$ BKM120 or equivalent dilutions ( $\mathrm{V} / \mathrm{v}$ ) of dimethylsulphoxide vehicle control for $48 \mathrm{~h}$. (d) BKM120 treatment reduces tumour cell viability with a half-maximal inhibitory concentration (IC 50 ) of $0.96 \pm 0.25 \mu \mathrm{M}$. Significant differences based on the average per cent inhibition \pm s.d. of three independent tumour cell lines treated with BKM120- versus vehicle-treated cells were calculated using a two-tailed Student's $t$-test $\left({ }^{\star} P\right.$ value $\left.<0.05\right)$. (e,f) Administration of chow-fed BKM120 reduces PI3K pathway activity, as shown by reduced P-S6 Ser235/236 levels, and extends the Arid1al/fl; (Gt)Rosa26Pik3ca ${ }^{*} H 1047 R$ median survival latency to 11 weeks. BKM120 treatment commenced 4 weeks after AdCRE injection. P-S6 Ser235/236 IHC was performed on tumour histological sections from three independent BKM120-treated or -untreated mice. (f) Untreated Arid19 ${ }^{f / f l} ;(G t) R o s a 26 P i k 3 c a^{*} H 1047 R$ mice succumb to cancer with a median ( $\left.\mu_{1 / 2}\right)$ latency of period of 7.5 weeks. Statistical differences in survival (BKM120 chow-fed versus untreated) were calculated using a log-rank test $(P$ values $<0.05$ were considered significant).

To further assess the similarities between our genetic model and human OCCC, we compared the mouse tumour-specific gene expression profiles for our Aridla ${ }^{f l / f l} ;$ (Gt)Rosa26Pik3ca ${ }^{*} H$ Ho47R mouse model to those recently reported for the Aridlatlff; Pten ${ }^{f l f l}$ mouse model by Guan $e t$ al. $^{26}$, which is genetically similar endometrioid EOC. Both models relied on the intrabursal AdCRE injection method for mutation induction. We applied principal component analysis using the normalized expression values for all genes in the tumour data sets for each mouse model. We found that each tumour sample clustered based on the genotype or mutational pattern of the respective genetic model, with the exception of one Aridla ${ }^{f l / f}$; Pten ${ }^{f l / f l}$ tumour (Supplementary Fig. 8). These findings are consistent with the marked phenotypic differences observed in our Arid1 $a^{l l f l} ;(G t)$ Rosa26Pik3ca ${ }^{* H 1047 R}$ tumours when compared with the phenotypes reported for Aridla ${ }^{f l / f l} ;$ Pten ${ }^{f l / f l}$ tumours by Guan et al. ${ }^{26}$ 


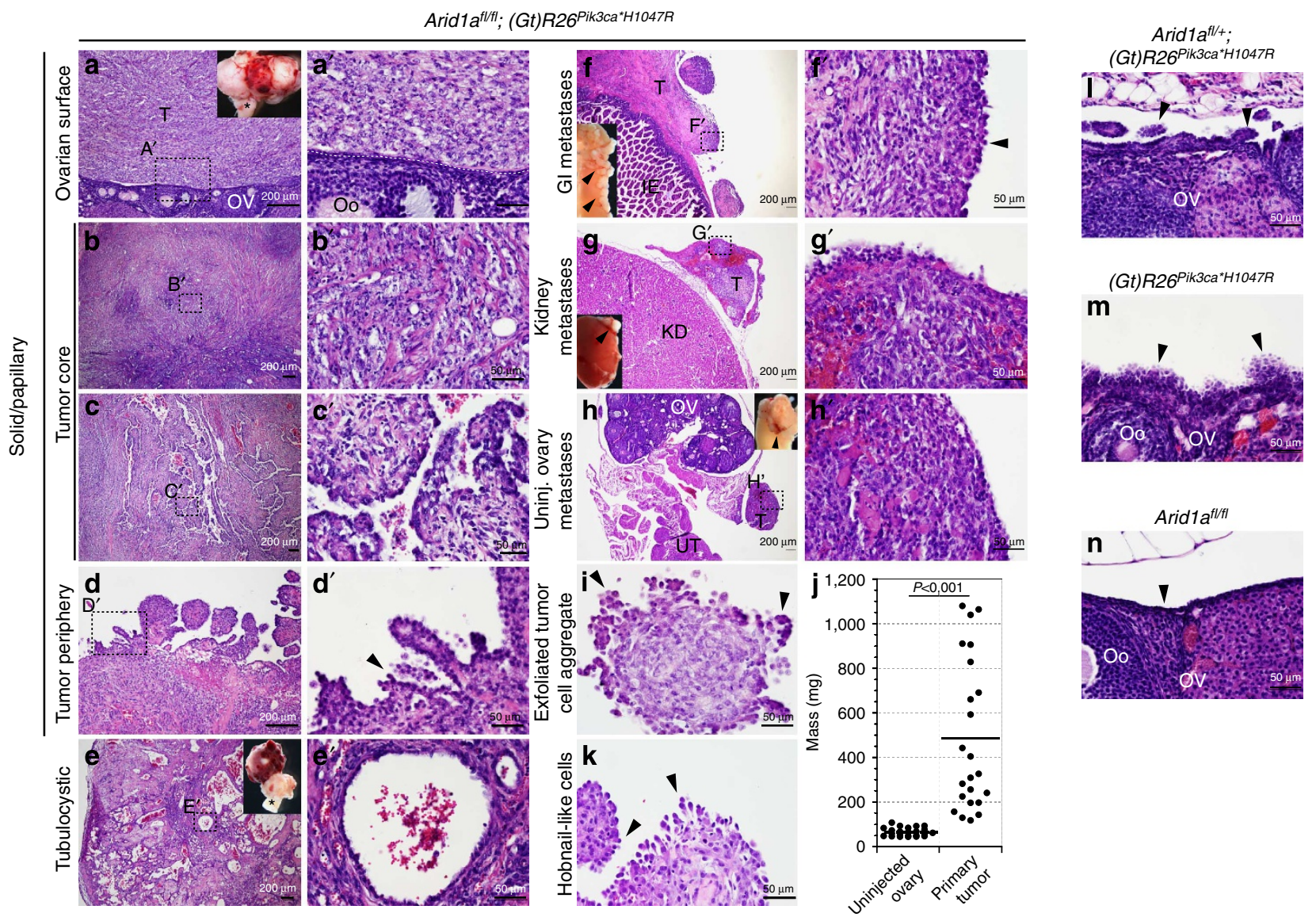

Figure 4 | Arid1a ${ }^{f / f l} ;$ (Gt)Rosa26Pik3ca ${ }^{\text {*H1047R }}$ ovarian tumours manifest clear-cell-like histological features. (a-e) Haematoxylin and eosin (H\&E)stained sections of tumour architectural patterns observed in intrabursal AdCRE-injected Arid1a ${ }^{f l / f l}$; (Gt)Rosa26Pik3ca ${ }^{*}$ H1047R mice. Whole-mount tumour images are shown inset in a,e. Asterisks denote the uterus. Dotted line $\mathbf{a}^{\prime}$ demarcates the ovarian surface-tumour boundary. Arrowhead in $\mathbf{d}^{\prime}$ denotes a hobnail cell. (f-h) H\&E-stained sections of Arid7 $a^{f / f l} ;(G t) R o s a 26 P i k 3 c a^{*} H 1047 R$ tumour metastases found on the surface of gastrointestinal tract (f), kidney (g) and uninjected, control ovary (h). ( $\left.\mathbf{a}^{\prime}-\mathbf{h}^{\prime}\right)$ High-magnification images of regions demarcated by dashed boxes in a-h. (i) H\&E-stained exfoliated tumour cell aggregate. (k) H\&E-stained example of hobnail cells on the luminal surface of a tumour. Arrowheads in $\mathbf{d}^{\prime}, \mathbf{f}^{\prime}, \mathbf{i}, \mathbf{k}$ denote hobnail cells on tumour surface. (j) Dot plot of primary tumour mass compared with uninjected control ovary. Significant differences based on the average mass \pm s.d. between tumours and matched uninjected ovaries were calculated using a two-tailed Student's $t$-test ( $P$ values $<0.05$ were considered significant). (I-n) H\&E-stained high-magnification images of the ovarian surface from Arid1all + ; (Gt)Rosa26Pik3ca ${ }^{*} H 1047 R$, (Gt)Rosa26Pik3ca ${ }^{*} H 1047 R$, and Arid17 ${ }^{f l f l}$ mice. (m,n) OSE hyperplasia (arrowheads) is observed in (Gt)Rosa26Pik3ca ${ }^{*} H 1047 R$ and Arid1a ${ }^{f l /}{ }_{;} ;(G t) R o s a 26 P i k 3 c a^{*} H 1047 R$ mice. IE, intestinal epithelium; KD, kidney; Oo, oocyte; OV, ovary; T, tumour; UT, uterus.

Autocrine IL-6 signalling promotes tumour cell growth. IL-6 is an inflammatory cytokine that triggers JAK/STAT3 signalling and has prominent roles in tumour cell growth and differentiation ${ }^{43,44}$. High levels of circulating IL- 6 in cancer patients are associated with a poor prognosis ${ }^{43,44}$. In addition to its increased expression in OCCC tumours, IL- 6 has been detected in patient serum and tumour-derived cell lines, which has led to its proposed use as a therapeutic target in OCCC $^{40-42}$. In support of our cross-species microarray comparisons, we verified IL-6 expression in primary tumours and peritoneal metastases of Arid1a ${ }^{f l / f l}$; $(\mathrm{Gt})$ Rosa26Pik3ca ${ }^{*} H 1047 R$ mice by RT-PCR (Fig. 6b). We also observed high levels of secreted IL- 6 in the body fluids of tumour-burdened mice, with $>2,000 \mathrm{pg} \mathrm{ml}^{-1}$ of IL-6 in ascitic fluid aspirates (Fig. 6c,d). Consistent with this, strong IL-6 immunoreactivity was also observed in the mouse tumours by IHC (Fig. 6e).

To understand the role of IL- 6 in OCCC pathogenesis, we treated primary Arid1afl/fl; (Gt)Rosa26Pik3ca ${ }^{* H 104 \% R}$ mouse ascites-derived tumour cells, isolated as described above, with anti-IL-6-neutralizing antibodies. Anti-IL-6 treatment reduced the tumour cell viability and downregulated the phosphorylation of STAT3 at tyrosine 705 (P-STAT3 Y705), suggesting that autocrine IL-6-STAT3 signalling promotes tumour cell growth (Fig. 6f,g). Next we generated primary mouse tumour cell lines that stably express IL-6 short hairpin RNAs (shRNA) for use in tumour growth assays (Fig. 6h,i). IL-6 shRNA-expressing cells showed reduced growth rates in culture, and media supplementation with recombinant IL- 6 restored the normal growth patterns of IL-6 shRNA-expressing cells (Fig. 6j). To further examine tumour cell autonomous roles for IL-6 in ovarian tumour cell growth, we grafted control shRNA- and IL-6 shRNA-expressing mouse tumour cells onto the flanks of immunodeficient nude mice. Remarkably, the ascitic tumour cell grafts were indistinguishable from the ovarian tumours with regards to histopathological features (for example, clear cytoplasm), despite being maintained in tissue culture and further grown under the skin of immunodeficient mice (Fig. 6k,l). The rate of tumorgraft growth for IL-6 shRNAexpressing cells was significantly less than control shRNAexpressing cells, further indicating that autocrine IL-6 signalling contributes to OCCC pathogenesis by promoting tumour cell growth (Fig. $6 \mathrm{~m})$. 

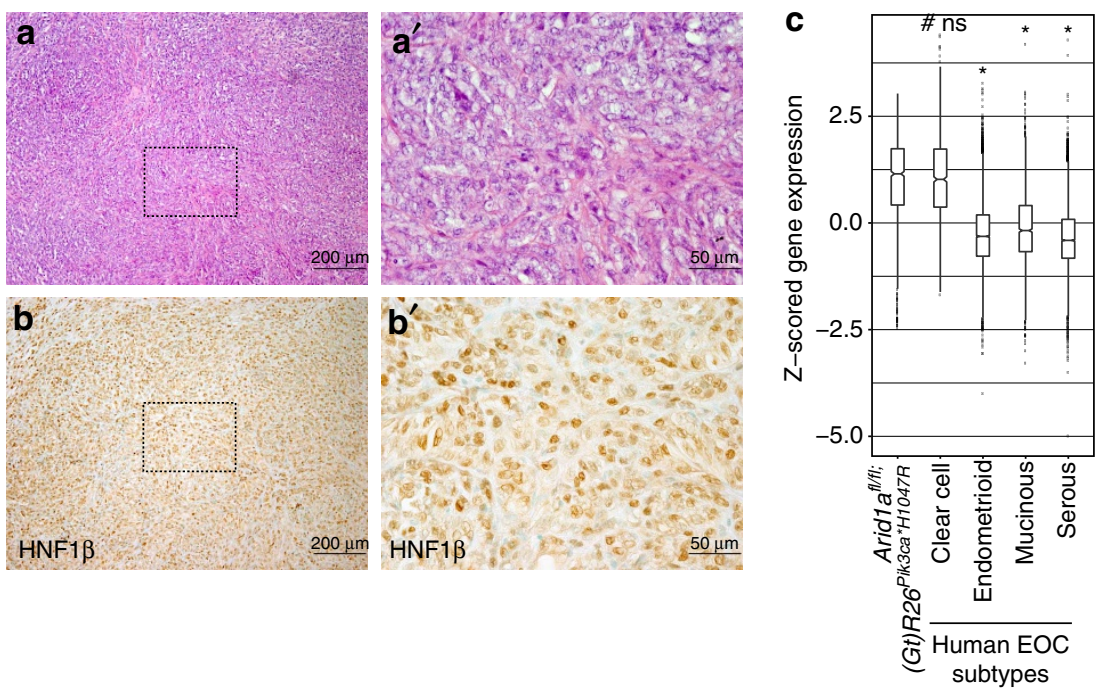

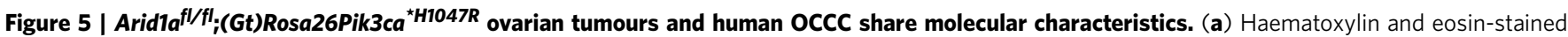
image of Arid1al/fl; (Gt)Rosa26Pik3ca ${ }^{*} H 1047 R$ tumour histology. (b) Near-adjacent histological section showing HNF1 $\beta$ immunoreactivity by IHC. $\left(\mathbf{a}^{\prime}, \mathbf{b}^{\prime}\right)$ High-magnification images of regions demarcated by dashed boxes in $\mathbf{a}, \mathbf{b}$. (c) Box plots of normalized expression values of human OCCC discriminant genes $(N=159)$ for all mouse tumour and human EOC subtype samples. Significant differences between the mouse tumours and each human histological EOC subtype were calculated using a Wilcoxon test ( ${ }^{*}$ significant $P$ value of $<0.05$; \#not significant $P$ value $=0.6$ ). NS, not significant.

IL-6 induction requires coexistent $A R I D 1 A-P I K 3 C A$ mutations. To determine if coexistent ARID1A-PIK3CA mutations are required for IL-6 induction, we utilized an AdCRE-inducible primary OSE cell culture model. IL-6 expression was measured following AdCRE infection of primary OSE cells isolated from mice carrying all mutant allele combinations. Loss of ARID1A or PIK3CA activation alone led to significant increases in IL-6 expression by RT-PCR and enzyme-linked immunosorbent assay (ELISA), and IL-6 induction was further enhanced when ARID1A and PIK3CA were co-mutated together in Aridla ${ }^{f l / f l}$; $(\mathrm{Gt})$ Rosa26$P i k 3 \mathrm{ca}^{{ }^{*} H 1047 R}$ cells (Fig. 7a,b). Consistent with this, P-STAT3 Y705 levels also correlated with similar increases in IL-6 activity in AdCRE-treated OSE cells (Fig. 7c). ARID1A loss in AdCREtreated Arid1 $a^{f l / f l}$ or Arid1a ${ }^{f l f l}$; (Gt)Rosa26Pik3ca ${ }^{*}$ H1047R OSE cells did not further enhance PI3K pathway activity (Fig. 7c). We found that Arid1 $a^{f l / f l}$; (Gt)Rosa26Pik3ca ${ }^{{ }^{*} H 1047 R}$ OSE cell proliferation was suppressed by treatment with anti-IL-6-neutralizing antibodies following mutation induction with AdCRE, and that recombinant IL-6 enhances normal OSE cell proliferation and IL-6-STAT3 signalling, further suggesting that IL-6 is both necessary and sufficient for tumour cell growth (Fig. 7d-f). To determine if ARID1A is bound at the IL-6 promoter under repressed conditions, we performed a series of ARID1A chromatin immunoprecipitation (ChIP) experiments in primary OSE cells. We used Arid1 $a^{f l / f l} ;(G t) R o s a 26 P i k 3 c a^{* H 1047 R}$ OSE cells for these experiments so that AdCRE-treated or ARID1A-depleted cells could be used as a control for ARID1A immunoprecipitation specificity. In AdControl-treated cells, increased ARID1A occupancy was observed at sites near the $I L-6$ promoter, but not at distal upstream or downstream sites (Fig. 7g). ARID1A occupancy at this site decreased in a manner consistent with ARID1A depletion following AdCRE infection. These data indicate that coexistent ARID1A-PIK3CA mutations lead to IL-6 upregulation.

\section{Discussion}

Our data support a genetic epistasis model wherein ARID1A and PIK3CA mutations cooperate and ovarian cancer can arise only when these genes are co-mutated in the mouse OSE. The short latency period, lack of copy-number variation and OCCC-like histological and molecular features we observed are also consistent with coexistent ARID1A-PIK3CA mutations being a major driver of OCCC in humans. Our results suggest that high levels of tumour cell-derived IL-6 are promoting tumour growth in OCCC. Indeed, the top pathway we identified in common between species is comprised of genes involved in this pathway, suggesting that IL-6-STAT3 signalling is inherent to OCCC biology. Hence, IL-6 serves as both a molecular marker and a prospective therapeutic target in OCCC. Therefore, anti-IL-6 antibody (Siltuximab) therapy may prove to be a safe and effective treatment strategy for OCCC patients. More broadly, our findings further demonstrate that the continued identification of tumour mutations (that is, TCGA projects) will allow us to identify potential functional relationships among recurrent mutational patterns or cancer pathways that would have otherwise gone unnoticed.

The OSE-derived tumour phenotypes we observed in our Arid1a $a^{f l f l}$; (Gt)Rosa26Pik3ca ${ }^{*} 1047 R$ mouse model of OCCC greatly contrasted with those reported by Guan et al. ${ }^{26}$ for Arid1 $a^{f l / f l}$; Pten fl/fl mice. For example, all of the tumour-burdened Arid1aflfl; (Gt)Rosa26Pik3ca ${ }^{*}$ H1047k mice were morbid or succumbed to death within 3 months after AdCRE injection, whereas Guan et al. ${ }^{26}$ reported tumours in a subset of Aridla fl/fl; Pten ${ }^{f l / f l}$ mice after 6 months, indicating stark differences in tumour latency or survival. Molecular differences were evident in tumour gene expression comparisons between the two mouse models, as each gene expression data set parsed the majority of tumour samples based on its respective mutational pattern or genotype. These data point to potential functional differences between ARID1A-PIK3CA and ARID1A-PTEN mutational patterns in Type I EOCs and support the idea of a possible PI3K-AKT-mTOR-independent role for PTEN in endometrioid EOC $^{26}$. Alternatively, it remains possible that clear-cell tumorigenesis is driven, in part, by an alternative AKTindependent mechanism downstream of oncogenic PIK3CA mutations ${ }^{45,46}$. Additional pharmacological studies targeting downstream effectors or alternative signalling pathway components will further address potential PI3K pathwayindependent mechanisms of endometrioid versus clear-cell tumorigenesis. 
a

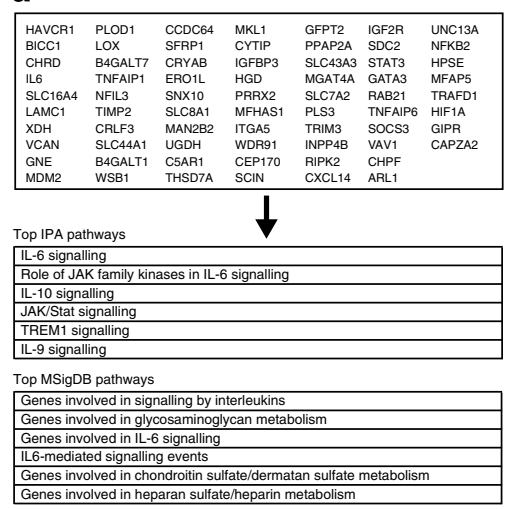

$f$

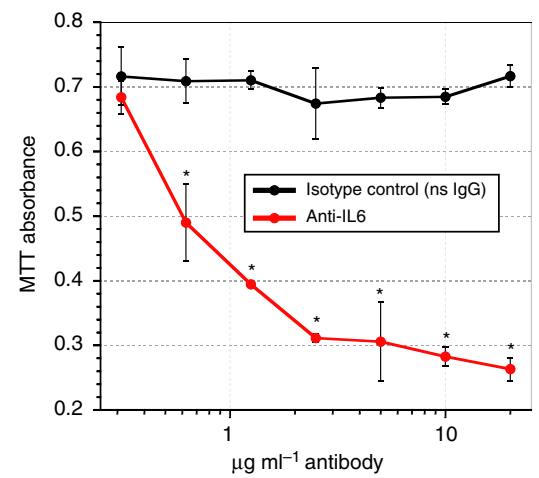

j

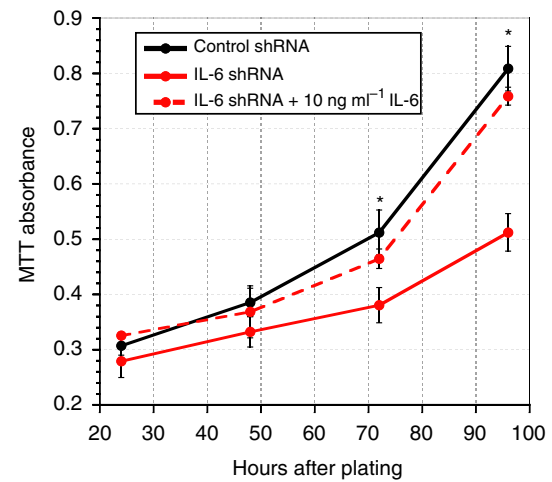

b

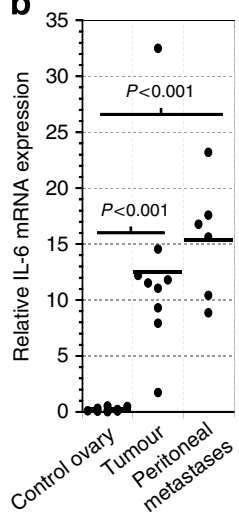

C

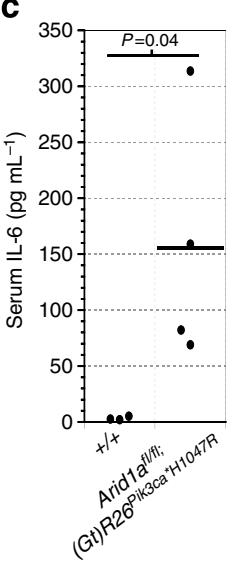

g

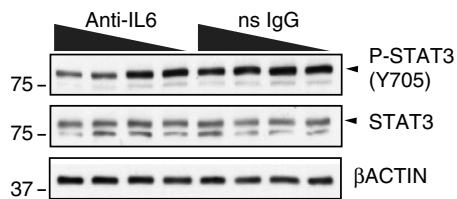

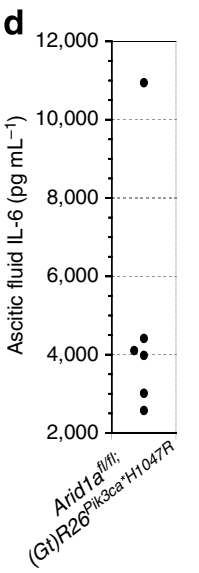

h

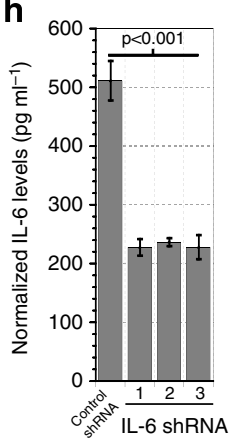

e

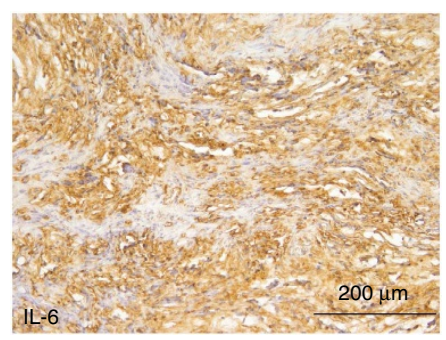

i

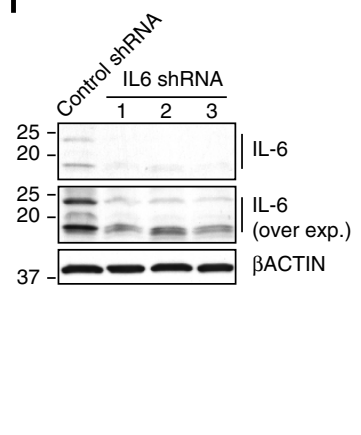

$\mathbf{k}$

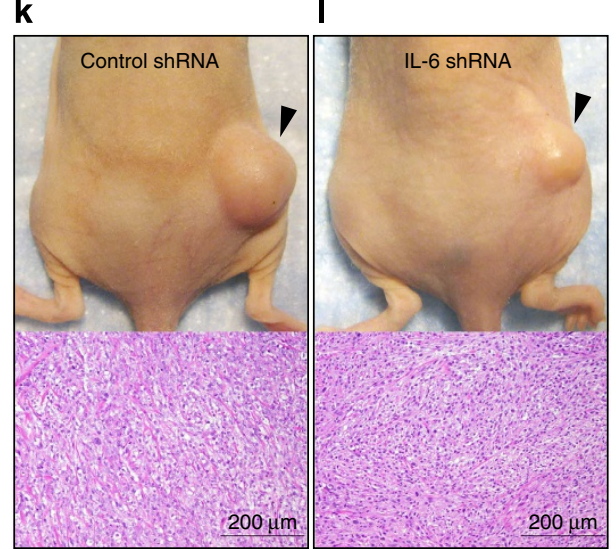

$\mathbf{m}$

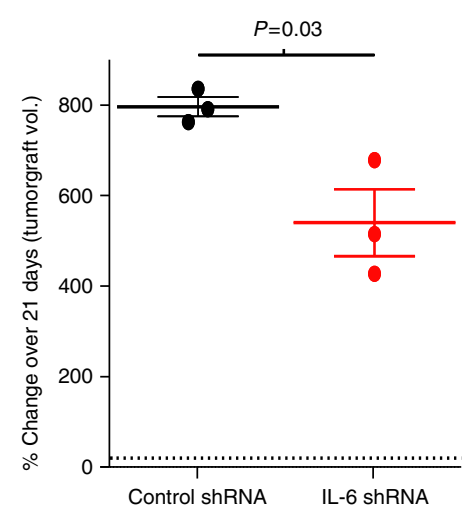

Figure 6 | Tumour-derived IL-6 promotes OCCC tumour cell growth and survival. (a) Top OCCC-specific genes in common between mouse and human tumours. Top six Ingenuity Pathway Analysis (IPA) and MSigDB (GSEA) predictions of the top upregulated genes are included. (b) RT-PCR validation of IL-6 expression in primary tumours and peritoneal metastases. Significant differences based on average normalized mRNA expression \pm s.d. between peritoneal metastases or primary tumours and matched uninjected ovaries were calculated using a two-tailed Student's $t$-test. (c,d) Mouse IL- 6 levels in the serum and ascitic fluid Arid1a ${ }^{f l / f l}$; (Gt)Rosa26Pik3ca ${ }^{\star} H 1047 R$ mice, as measured by anti-IL-6 ELISAs. Significant differences based on the average protein concentration \pm s.d. between wild-type versus Arid1al/fl; $(G t) R o s a 26 P i k 3 c a^{* H 1047 R}$ mice were calculated using a two-tailed Student's $t$-test. (e) IL-6 expression in an Arid1a ${ }^{f l / f l}$; (Gt)Rosa26Pik3ca ${ }^{*}$ H1047R tumour by IHC. (f) MTT absorbance values plotted with log antibody concentration ( $\mu$ g ml ${ }^{-1}$ ) for non-specific rat IgG-treated (control) or rat anti-mouse IL-6-treated Arid19 ${ }^{f l / f l}$; (Gt)Rosa26Pik3ca ${ }^{\star} H 1047 R$ ascitic tumour cells after $96 \mathrm{~h}$. of treatment. Plot represents the average absorbance value \pm s.d. for treatment performed on three independent cell lines. Significant differences between control- and anti-IL-6-treated cells were calculated using a two-tailed Student's $t$-test ( ${ }^{*}$ significant $P$ value $<0.05$ ). (g) Primary ascitic tumour cells were treated 10,1 , 0.01 or $0 \mu \mathrm{g} \mathrm{ml}^{-1}$ rat anti-mouse IL-6 or non-specific Rat IgG-treated (control). (h,i) IL-6 expression in Arid7al/fl; (Gt)Rosa26Pik3ca ${ }^{*}$ H1047R ascitic tumour cells stably expressing control shRNAs or IL- 6 shRNAs by ELISA and western blot. Each replicate represents in stable pool of IL-6 shRNA-expressing cells from three independently isolated tumour cell lines. Significant differences based on the average protein concentration $\pm \mathrm{s}$.d. between control versus IL-6 shRNA were calculated using a two-tailed Student's $t$-test. (j) MTT absorbance values plotted over time for control shRNA-, IL-6 shRNA- or IL-6 shRNA-expressing cells supplemented with $10 \mathrm{ng} \mathrm{ml}^{-1} \mathrm{IL}-6$. Cells were plated at $2 \times 10$ e 4 cells $\mathrm{ml}^{-1}$ at hour 0 , then MTT measurements were taken every $24 \mathrm{~h}$ for a total of $96 \mathrm{~h}$. Significant differences between control- and IL- 6 shRNA-expressing cells were calculated using a two-tailed Student's $t$-test ( ${ }^{*}$ significant $P$ value $<0.05$ ). (k,I) Whole-mount images of control- and IL-6 shRNA tumorgrafts (arrowheads) on the right flank of nude mice and images of corresponding haematoxylin and eosin-stained tumorgraft sections. (m) Plot of growth rates for control- and IL-6 shRNA-expressing tumorgrafts over 21 days of measurement. Significant differences between control- and IL-6 shRNA tumorgraft growth rates were calculated using a two-tailed Student's $t$-test. Only $P$ values $<0.05$ were considered significant. MTT, 3-(4,5-dimethylthiazol-2-yl)-2,5-diphenyltetrazolium bromide. 
a

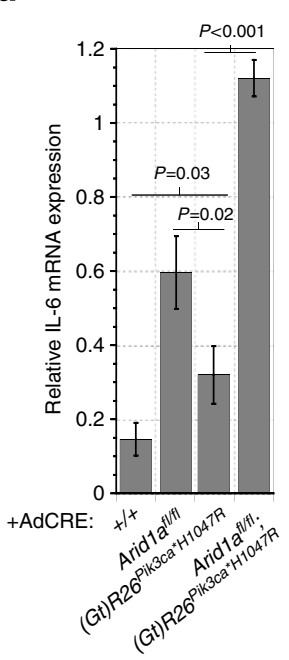

b

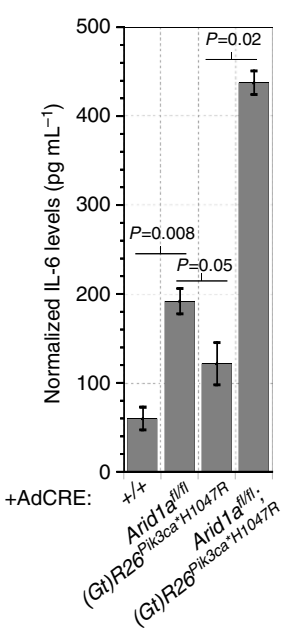

c

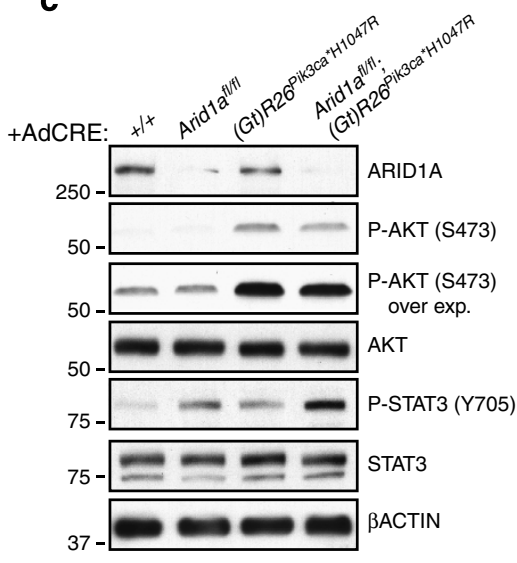

d

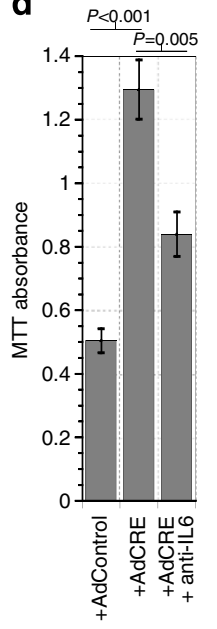

e

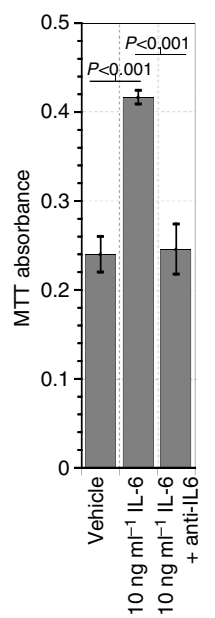

f

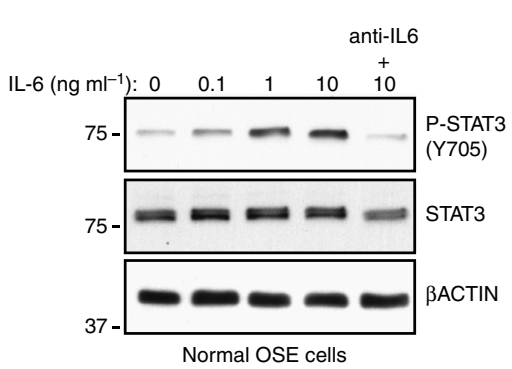

g

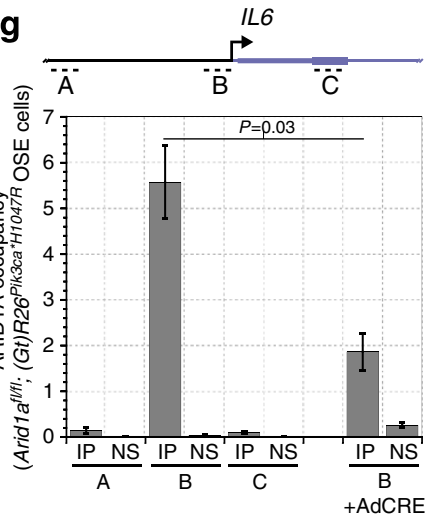

h

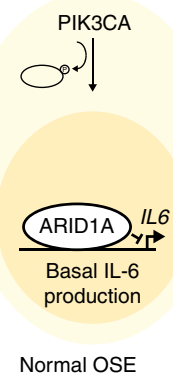

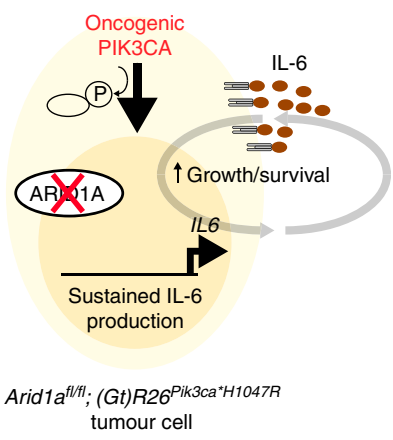

Figure 7 | Coexistent ARID1A-PIK3CA mutations induce IL-6 expression in a cooperative manner. (a) IL-6 mRNA expression in AdCRE-infected primary OSE cells carrying all allele combinations. (b) Normalized IL-6 ELISA measurements of AdCRE-infected primary OSE cells carrying all allele combinations. Significant differences based on the average normalized expression value \pm s.d. among mutant allele combinations versus wild type were calculated using a two-tailed Student's $t$-test. (c) Western blot showing ARID1A, P-AKT S473, total AKT, P-STAT3 Y705 and total STAT3 levels in AdCRE-infected primary OSE cells carrying all allele combinations. AdCRE-infected wild-type cells served as controls. (d) MTT cell viability assay on primary Aridla ${ }^{f l / f l}$;

(Gt)Rosa26Pik3ca ${ }^{*}$ H1047R OSE cells treated with AdControl, AdCRE or AdCRE supplemented with $5 \mu \mathrm{g} \mathrm{ml}^{-1}$ anti-IL-6 antibodies. (e) Treatment of normal (wild-type) primary OSE cells with vehicle ( $\mathrm{V} / \mathrm{v}), 10 \mathrm{ng} \mathrm{ml}^{-1} \mathrm{IL}-6$ or $10 \mathrm{ng} \mathrm{ml}^{-1} \mathrm{IL}-6$ supplemented with $5 \mu \mathrm{ml}^{-1}$ anti-IL-6 antibodies. Significant differences based on the average MTT absorbance value \pm s.d. between three independent replicates of AdControl- versus AdCRE-infected or vehicleversus IL-6-treated cells were calculated using a two-tailed Student's t-test. (f) Western blot of normal primary OSE cells showing dose-dependent increases in P-STAT3 Y705 levels following IL-6 treatment. Co-treatment with $5 \mu \mathrm{g} \mathrm{ml}{ }^{-1}$ anti-IL-6 antibodies blocked P-STAT3 Y705 induction. (g) ARID1A occupancy at the IL-6 locus was detected by ChIP using anti-ARID1A antibodies (denoted as IP) on crosslinked chromatin from AdControl- or AdCREinfected Arid1a ${ }^{f l / f l}$; (Gt)Rosa26Pik3ca ${ }^{*}$ H1047R primary OSE cells. Non-specific isotype-matched antibodies (denoted as NS) were used in control ChIPs.

ARID1A occupancy at the IL-6 promoter (site or primer pair $\mathbf{b}$ ) is reduced in ARID1A-depleted cells treated with AdCRE. Average per cent ChIP input \pm s.d. represent data from experiments performed using three primary OSE cell isolations. Significant differences were calculated using a two-tailed Student's t-test. (h) Proposed model of IL-6 regulation by ARID1A and PIK3CA mutations in OCCC. Only P values $<0.05$ were considered significant.

Several theories on the origins of OCCC have been proposed. Our data strongly suggest that the OSE harbours a cancer-prone cell population that gives rise to OCCC-like tumours in the mouse, which is consistent with the classical view of EOC pathogenesis. Nonetheless, our mouse model does not address the role of distal endometriotic explants or other putative precursor lesions in OCCC pathogenesis. Specifically targeting the ARID1A-PIK3CA mutational pattern to other tumour-prone epithelia within the Müllerian duct system would add relevance to our findings and help to further clarify the origins of EOC. An improved set of inducible CRE alleles would facilitate these experiments.

Inflammation of coelomic epithelial cell derivatives in the female reproductive tract is thought to be a major contributor to malignant transformation in endometriosis-associated EOC, like
OCCC $^{5,47}$. Accordingly, inflammatory mediators, aberrant immune modulation or irregular reproductive hormone levels are thought to play a major role in malignant transformation ${ }^{5,47}$. Inflammatory cytokine signalling pathways, like IL-6, may be part of the natural inflammatory repair process accompanying each ovarian and menstrual cycle that, when left unchecked, promotes unregulated epithelial cell growth. In support of this, our data demonstrate that OSE cells are competent to respond to and upregulate IL-6. Therefore, the incomplete resolution of epithelial cell inflammation during these repair processes may represent a common mechanism underlying OCCC tumorigenesis, irrespective of mutational pattern.

The identification of IL-6 as a physiological target of ARID1A tumour-suppressor activity in our OCCC tumour model raises many important questions regarding the role of 
ARID1A-containing SWI/SNF complexes in normal coelomic epithelial cell homeostasis. Indeed, ARID1A mutations have been identified in a wide variety of cancers originating from coelomic epithelial derivatives throughout the gynaecologic tract. Moreover, loss of ARID1A immunoreactivity is observed in endometriosis, a disease intimately associated with inflammation ${ }^{48-50}$. Endometriosis is also characterized by increased IL- 6 expression, but the relationship between ARID1A loss and IL- 6 upregulation in endometrial tissue is unknown ${ }^{51,52}$. Thus, ARID1A may regulate coelomic epithelial cell homeostasis and prevent tumorigenic conversion by negatively regulating the inflammatory programs required for normal tissue repair during the female reproductive cycle. It will be interesting to know if reproductive hormones influence ARID1A activity in this regard. Additional work on the propensity of ARID1A mutant epithelial cells to undergo malignant transformation in response to inflammatory 'insults' or the role of key inflammatory response pathways in this process will be required to address these hypotheses. Thus, the loss of ARID1A-containing SWI/SNF complexes might expose the 'darker,' pro-tumorigenic side of inflammation.

How do oncogenic PI3K signalling mutations contribute to this process or lead to increased IL-6 expression? Positive regulators of IL-6 include JAK/STAT, PI3K, EGFR/HER2, RAS/RAF/MEK, TNF/TLR/IL1 or RAS/ERK oncogenic pathways and AP-1, SP1, NF- $\kappa B, C / E B P, S T A T 3$, and CREB transcription factors ${ }^{53}$. IL-6-mediated JAK/STAT activation can further promote IL-6 transcription ${ }^{53}$. We propose that oncogenic PIK3CA ${ }^{H 1047 R}$ mutations act like inflammatory 'insults' and drive the signalling loop that sustains high levels of IL-6 production in the absence of negative regulation by ARID1A (Fig. 7h). Additional studies focusing on the intricacies of this feedforward signalling loop and the potential crosstalk mechanisms occurring among the various pathways that sustain it will further explain why coexistent ARID1A-PIK3CA mutations are found in a wide variety of human cancers.

\section{Methods}

Generation of the Arid1a conditional mouse allele. The Arid1all conditional mutant mouse allele was engineered using the bacterial artificial chromosome recombineering methods developed by Liu et al. ${ }^{54}$ Correctly targeted mouse embryonic stem cell clones were identified by Southern blotting. The Neo selection cassette used for targeting was removed by FLP-mediated recombination ${ }^{54}$. Targeted embryonic stem cells were injected into C57Bl6 blastocysts and implanted into pseudopregnant females. Germline animals were detected by coat colour and PCR confirmation of tail DNA samples.

\begin{abstract}
AdCRE-mediated genetic recombination in the ovary. To induce genetic recombination in the OSE, we employed a modified version of the ex vivo Adenovirus CRE (AdCRE) intrabursal delivery method ${ }^{7,29}$. In brief, AdCRE particles (obtained from the University of Iowa Gene Transfer Core) were diluted in sterile $1 \times$ Dulbecco's PBS (dPBS) containing $8 \mu \mathrm{g} \mathrm{ml}^{-1}$ polybrene. Deeply anaesthetized 8-10-week-old mice were given a single $5 \mu$ linjection of AdCRE particles $\left(2.5 \times 10^{7}\right.$ plaque-forming units or p.f.u.) into the right ovarian bursal cavity of the surgically exposed ovary using a sterile 31 -gauge needle. Immediately following AdCRE injection, the surgically excised ovaries were washed with sterile $1 \times$ dPBS and placed back into the abdominal cavity. The surgeries were performed on multiple occasions. Age- and genotype-matched, non-littermate animals were randomized before AdCRE injection. The surgeon was blinded to the genotypes before surgery and AdCRE injection. All surgical procedures were performed in accordance with protocols approved by the University of North Carolina at Chapel Hill Institutional Animal Care and Use Committee.
\end{abstract}

\footnotetext{
Mouse husbandry and genotyping. All mice were maintained on an outbred (random) genetic background using CD-1 mice (Charles River). (Gt)ROSA26Pik3ca*H1047R and Sox2CRE alleles were purchased from the Jackson Laboratory and identified by PCR using published methods ${ }^{28,35}$. Arid1 $a^{+/+} ;$Arid1 $a^{f l /+} ;$ Arid $1 a^{f l / f l}$ alleles were distinguished by PCR using the following primer set: (F) $5^{\prime}$-CTAGGT GGAAGGTAGCTGACTGA-3'; (R) 5' -TACACGGAGTCAGGCTGAGC-3'. Combining the forward primer (from above) with the following reverse primer
} amplifies the CRE-excised allele: (R) 5' -AGAGTAACTAATAACTGCTGGAGG
ATG- $3^{\prime}$. PCR products were resolved by $2 \%$ Tris-Borate-EDTA agarose gel electrophoresis. End points were palpable tumours $\left(>1.5 \mathrm{~cm}^{3}\right)$, visible haemorrhagic ascites or severe abdominal distension, and signs of severe illness, such as dehydration, hunching, ruffled fur, signs of infection or nonresponsiveness. Sample sizes within each genotype were chosen based on the proportions of animals with tumours and/or survival between each experimental group or a Kaplan-Meier log-rank test for survival differences. Whole-mount liveanimal luminescent imaging was performed using the IVIS Optical System and reagents (Perkin Elmer) according to the manufacturer's instructions. Wholemount images of mice or necropsies were acquired using a Canon PowerShot SD1100 IS digital camera and a tabletop tripod. Whole-mount tumour specimens were imaged on a Leica MZ FLIII stereomicroscope using a DS Ril digital camera (Nikon). All mice were maintained at the University of North Carolina at Chapel Hill, Animal Facility using standard techniques in accordance with protocols approved by the University of North Carolina at Chapel Hill Institutional Animal Care and Use Committee.

In vivo drug treatments. The pan-PI3K inhibitor, BKM120 (Novartis) was milled by Research Diets using a vehicle- or carrier-free formulation similar to standard husbandry diets and dosed at $40 \mathrm{mg} \mathrm{kg}^{-1}$ based on the average daily oral consumption of a mouse ${ }^{55-57}$. AdCRE-injected animals were randomized before drug treatment. Control or untreated mice were given a standard husbandry diet. All drug treatments were performed in accordance with protocols approved by the University of North Carolina at Chapel Hill Institutional Animal Care and Use Committee.

Reverse transcription-PCR. Total RNA was extracted from pulverized tumour samples or ovarian epithelial cells using the TRIzol method (Invitrogen), followed by a RNA cleanup step and on-column DNA digestion using the RNAeasy mini prep kit (Qiagen) according to the manufacturers' instructions. To analyse ARIDIA and (Gt)Rosa26 $6^{P i k 3 c a^{*} H 1047 R}$ gene transcript levels, real-time quantitative PCR was performed using Ssofast PCR master mix (Biorad) and a CFX96 thermocycler (Biorad) with the following gene-specific primers: ARID1A (F) 5 -CTGAAGGA CAAAGGTGACT-3', ARID1A (R) 5'-CACAACTGCGAACTTCTC-3'; R26exon1 (F) 5'-CTAGGTAGGGGATCGGGACTCT- ${ }^{\prime}$, PIK3CAcDNA (R) $5^{\prime}$-AATTTCTC GATTGAGGATCTTTTCT-3' (ref. 35). The following gene-specific primers were used to validate the microarray expression data: KRAS (F) $5^{\prime}$-AGAGGAGTACAG TGCAATGAGG-3', KRAS (R) 5'-AGGCACATCTTCAGAGTCCTTT-3'; TFPI2 (F) 5'-AAGGGCTTGTGTGAACCACG-3', (R) 5'-CCACCACAGCCAGTATAG GTG-3'; CDKN1A (F) 5'-ATCCAGACATTCAGAGCCACAG-3' (R) 5' -ACGAA GTCAAAGTTCCACCGT-3'; CDKN2A (F) 5'-GCTTCTCACCTCGCTTGTCA-3' (R) $5^{\prime}$-AGTGACCAAGAACCTGCGAC- $3^{\prime}$; MUC16 (F) $5^{\prime}$-CTCATCTGCTTGG CGGTACT-3'; (R) 5'-GAACCCTGCTAGGGAAGAGC-3'; VCAN (F) $5^{\prime}$-GAA GGGAACAGTTGCTTGCG-3', (R) 5'-TTAGGCATTGCCCATCTCCC-3'; IL-6 (F) 5'-CACTTCACAAGTCGGAGGCT-3', (R) 5'-CTGCAAGTGCATCATCGT TGT- $3^{\prime}$. The expression was calculated from three independent experiments using $\Delta \Delta^{\mathrm{Ct}}$ methods. Expression levels were normalized to $\beta A C T I N$. Statistical differences were detected using a two-tailed Student's $t$-test. The following H1047 spanning PCR primer pair was used to distinguish endogenous PIK3CA from Rosa26-derived, PIK3CA ${ }^{H 1047 R}$ gene transcripts: (F) $5^{\prime}$-TTCAATGATGCTTGGCT CTG-3'; (R) 5'-CTGCTTGATGGTGTGGAAGA-3'. The PCR products were digested with MslI and resolved by $2 \%$ Tris-Borate-EDTA agarose gel electrophoresis.

Western blotting. Western blots were performed using the following antibody dilutions and standard chemiluminescent detection methods or Li-COR Bioscience Odyssey fluorescent western blotting reagents: 1:1,000 ARID1A (A301-041A, Bethyl Labs,), 1:500 IL-6 (ab6672, Abcam), 1:1,000 Phospho-AKT Ser473 (4060, Cell Signaling), 1:1,000 AKT (4691, Cell Signaling), 1:100 STAT3 (sc-482, Santa Cruz Biotechnology), 1:1,000 Phospho-STAT3 Tyr705 (9145, Cell Signaling) and 1:5,000 $\beta$ ACTIN (Abcam). Membranes were blocked in $1 \times$ PBS supplemented with $5 \%(\mathrm{w} / \mathrm{v})$ non-fat dry milk (Blotto A) or $1 \times$ Tris-buffered saline $(\mathrm{pH} 7.6)$ supplemented with $5 \%(\mathrm{w} / \mathrm{v})$ bovine serum albumin (BSA). Primary antibody incubations were performed in $1 \times$ PBS supplemented with $1 \%(\mathrm{w} / \mathrm{v})$ non-fat dry milk, $1 \%(\mathrm{w} / \mathrm{v})$ BSA and $0.05 \%(\mathrm{v} / \mathrm{v})$ Tween-20 (Blotto B) or $1 \times$ Tris-buffered saline ( $\mathrm{pH} 7.6)$ supplemented with $5 \%(\mathrm{w} / \mathrm{v})$ BSA. Western blot images have been cropped for presentation. Full-size western blot images with antibody dilutions and blocking buffer conditions are presented in Supplementary Figs 9 and 10.

Enzyme-linked immunosorbent assay. IL-6 ELISAs were performed using the OptEIA mouse IL-6 kit reagents (BD Bioscience) according to the manufacturer's instructions. ELISA measurements were normalized to total cellular protein.

Copy-number and TCGA computational analyses. Tumour and matched control DNA samples were processed for hybridization on the high-density Mouse Universal Genotyping SNP Array (MegaMUGA, Neogen) using published methods ${ }^{58}$. Copy-number analysis was performed using ASCAT ${ }^{59}$. ARID1A 
and/or PIK3CA TCGA mutation frequency was identified by querying the cBioPortal on 2014-10-21 using the R API ${ }^{60,61}$.

\section{Microarray and cross-species gene expression comparisons. Total RNA} was isolated from nine primary tumour specimens and nine matched normal (uninjected) ovaries as described above. Total RNA (250 ng) was used to synthesize fragmented and labelled sense-strand cDNA and hybridize onto Affymetrix Mouse Gene 2.1 ST peg plate arrays. The mouse data were uploaded to the Gene Expression Omnibus (GEO) under the accession number: GSE57380. Affymetrix CEL files were normalized using the Robust Multichip Average normalization method $^{62}$. Expression changes (tumour versus control ovary) were determined using Linear Models for Microarray Data Analysis ${ }^{63}$ or Significance Analysis of Microarray analysis ${ }^{64}$. Probes with a false discovery rate of $0 \%$ were considered statistically significant. For mouse-human cross-species comparisons, expression data for human OCCC and normal ovaries were downloaded from GEO under the accession number GSE6008 (ref. 39). Heat maps and dendrograms were generated with Java TreeView or R (www.R-project.org). Histological subtype-specific gene signatures were derived by first identifying differentially expressed genes between human normal tissue and cancer tissue in GSE6008. Next, differentially expressed genes were identified comparing each histological subtype to all other histological subtypes. We considered only upregulated genes, and any genes that were upregulated in both the tumour versus normal comparison and in a particular subtype were removed because these are not specific to a histological subtype and may reflect generic pan-cancer gene expression patterns. To compare human and mouse samples from these gene sets, we first determined $Z$-scores for each gene within the mouse or human. We combined these $Z$-scores and $Z$-scored the combined data using all human and mouse tumour samples in GSE6008 and this study. Pathways analysis was performed using the GSEA Molecular Signatures Database (MSigDB) ${ }^{65,66}$ or Ingenuity Pathway Analysis (Qiagen).

Ascites-derived tumour cell and OSE cell culture. Primary ascites-derived ovarian tumour cells were isolated and grown as previously described ${ }^{67}$. To facilitate complete red blood cell lysis, the cell isolates were incubated and washed in deionized $\mathrm{H}_{2} \mathrm{O}$ before resuspension in growth medium. Mouse OSE cells were isolated and cultured as previously described ${ }^{68}$. To induce CRE-mediated recombination in OSE cells, the cells were infected with AdCRE or AdControl particles (obtained from the University of Iowa Gene Transfer Core) at an MOI of 200 for $2 \mathrm{~h}$ in serum-free media. Infected OSE cells were then placed in normal growth medium and allowed to recover for 3 days before further manipulation. BKM120 (Chemitek), lowendotoxin rat anti-mouse IL-6-neutralization antibody (R\&D Systems, MAB406), non-specific rat $\operatorname{IgG}_{1}$ isotype control antibody (R\&D Systems, MAB005) or recombinant mouse IL-6 protein (R\&D Systems, 406-ML-005) were reconstituted according to the manufacturers' instructions and used as described in the text. For all antibody neutralization experiments, the cells were refed daily with the appropriate concentrations of antibody. Primary ascites-derived ovarian tumour cells were stably transduced with Mission pLKO.1-puro IL-6 shRNA (Hairpin sequence: 5'-CCGGCCAGAGTCCTTCAGAGAGATACTCGAGTATCTCTCTGAAG GACTCTGGTTTTTG-3') (Sigma, TRCN0000067551) or non-target control shRNA (Sigma, SHC016V) Lentiviral transduction particles according to the manufacturers' instructions. The MTT assay kit (Roche) was used to measure cell viability. Ascitesderived tumour cells and OSE cells were passaged using Accutase (Invitrogen). Halfmaximal inhibitory concentrations $\left(\mathrm{IC}_{50}\right.$ ) were calculated by curve-fitting using Kaleidagraph software (Synergy).

Tumour grafts. Primary ascites-derived ovarian tumour cells expressing controlor IL- 6 shRNAs were harvested by Accutase digestion and washed in $1 \times \mathrm{dPBS}$. The cells were then injected subcutaneously into the right flanks of immunodefi-

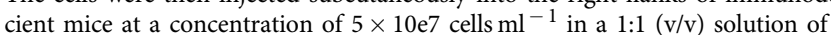
$1 \times$ dPBS and Cultrex basement membrane extract (3432-005-01, Trevigen). Tumour graft volumes were calculated using biweekly caliper-based measurements and growth rates were based on these volumetric measurements. All tumour grafts were performed in accordance with protocols approved by the University of North Carolina at Chapel Hill Institutional Animal Care and Use Committee.

Histology and IHC. For indirect IHC of slides, 10\% neutral-buffered formalin-fixed paraffin sections were processed for heat-based antigen unmasking in $10 \mathrm{mM}$ sodium citrate ( $\mathrm{pH}$ 6.0). Sections were incubated with the following antibody dilutions: 1:50 ARID1A (Clone PSG3, sc-32761, Santa Cruz Biotechnology), 1:100 Cytokeratin 8 (TROMAI, DSHB), 1:50 phospho-AKT Ser473 (4060, Cell Signaling), 1:50 phosphoS6 Ribosomal Protein Ser235/236 (4858, Cell Signaling), 1:100 HNF1 $\beta$ (sc-7411, Santa Cruz Biotechnology) or 1:400 IL-6 (ab6672, Abcam). Vectastain Mouse-onMouse blocking reagents (Vector Labs) were used for ARID1A IHC. SignalStain antibody diluent (8112, Cell Signaling) and SignalSlide AKT controls (8115, Cell Signaling) were used for phospho-AKT Ser473 and phospho-S6 Ribosomal Protein Ser235/236 IHC. Secondary detection was performed using Vectastain ABC System reagents (Vector Labs). Sections processed for IHC were lightly counterstained with Haematoxylin QS or Methyl Green (Vector Labs). Routine haematoxylin and eosin staining of sections was performed by University of North Carolina, Lineberger Cancer Center, Animal Histopathology Core. Histological tumour assessments were reviewed by a UNC animal pathologist. Histological sections were analysed and photographed on an Olympus BX51 microscope using bright-field optics and a DP series digital camera (Olympus).

Chromatin immunoprecipitation. Primary OSE cells were fixed in growth media containing $1 \%$ methanol-free formaldehyde (Pierce) for $10 \mathrm{~min}$ at room temperature. The cells were washed twice with $1 \times \mathrm{dPBS}$ and then swelled in cell lysis buffer ( $5 \mathrm{mM}$ Tris-Cl (pH 8.0); $85 \mathrm{mM} \mathrm{KCl;} \mathrm{1 \%} \mathrm{IGEPAL} \mathrm{CA-630)} \mathrm{for} 10 \mathrm{~min}$ on ice. Swollen cells were homogenized (20 strokes) with a B-type dounce homogenizer on ice to facilitate nuclei release. Isolated nuclei corresponding to $1 \times 10 \mathrm{e} 7$ cell equivalents per $500 \mu \mathrm{l}$ of nuclear lysis buffer $(50 \mathrm{mM}$ Tris- $\mathrm{Cl}(\mathrm{pH} 8.0), 10 \mathrm{mM}$ EDTA and $0.3 \%$ SDS) and then sonicated using a water bath-cooled Diagenode Biorupter sonicator and $1.5 \mathrm{ml}$ TPX microtubes ( $200 \mu \mathrm{l}$ sample volume per tube) to an average DNA length between 300 and $700 \mathrm{bp}$. Chromatin concentrations were determined using a BCA protein assay kit (Pierce). Chromatin samples were diluted to $300 \mu \mathrm{g}$ per $200 \mu \mathrm{l}$ of nuclear lysis buffer and then cleared by centrifugation at $10,000 \mathrm{~g}$. Precleared chromatin samples $(200 \mu \mathrm{l}$ each) were added to $800 \mu \mathrm{l}$ (1:5 dilution) of ChIP dilution buffer ( $50 \mathrm{mM}$ Tris-Cl (pH 7.5); $150 \mathrm{mM}$ $\mathrm{NaCl} ; 5 \mathrm{mM}$ EDTA; 0.5\% IGEPAL CA-630; 1\% TX-100) and incubated with $10 \mu \mathrm{g}$ of prebound ARID1A (Clone PSG3, 04-080, Millipore) Protein G dynal beads (Invitrogen) overnight at $4{ }^{\circ} \mathrm{C}$. The Protein $\mathrm{G}$ dynal bead-anti-ARID1A immune conjugates were washed twice with $1 \mathrm{ml}$ ChIP dilution buffer, three times with $1 \mathrm{ml}$ ChIP dilution buffer supplemented with $500 \mathrm{mM} \mathrm{NaCl}$, twice with $1 \mathrm{ml} \mathrm{ChIP}$ dilution buffer, followed by a final wash in $1 \mathrm{ml}$ low-salt TE buffer $(10 \mathrm{mM}$ Tris-Cl ( $\mathrm{pH} 8.0$ ); $1 \mathrm{mM}$ EDTA; $50 \mathrm{mM} \mathrm{NaCl}$ ). The immunoprecipitated samples were eluted in $100 \mu \mathrm{l}$ of elution buffer $\left(50 \mathrm{mM} \mathrm{NaHCO}_{3} ; 1 \% \mathrm{SDS}\right)$ at $62^{\circ} \mathrm{C}$ for $20 \mathrm{~min}$ using an Eppendorf tube mixer. The crosslinks were reversed by adding $\mathrm{NaCl}$ to a final concentration of $0.2 \mathrm{M}$ followed by an overnight incubation at $65^{\circ} \mathrm{C}$. The samples were then deproteinated with Proteinase $\mathrm{K}$ and purified using the ChIP DNA Clean \& Concentrator kit (Zymo Research) according to the manufacturer's instructions. Real-time quantitative PCR using Ssofast PCR master mix (Biorad) and a CFX96 thermocycler (Biorad) was performed using the following genespecific primer pairs: (IL-6, Site A) 5'-GCAACTCTCACAGAGACTAAAGG-3', $5^{\prime}$-GGACAACAGACAGTAATGTTGC-3' (ref. 69); (IL-6, Site B) 5' -CTAGCCTC AAGGATGACTTAAGC- $3^{\prime}, 5^{\prime}$-CTATCGTTCTTGGTGGGCTCCAGAGC- $3^{\prime}$ (ref. 70); (IL-6, Site C) 5'-GCCTTCTTGGGACTGATGCT-3', 5'-GACAGGTCT GTTGGGAGTGG-3'. Data from three independent primary OSE cell isolations were averaged and plotted as a percentage of the total input.

\section{References}

1. Siegel, R., Naishadham, D. \& Jemal, A. Cancer statistics, 2013. CA Cancer J. Clin. 63, 11-30 (2013)

2. Adashi, E. Y. \& Leung, P. C. K. The Ovary 591-602 (Elsevier Academic Press, 2004).

3. Perets, R. et al. Transformation of the fallopian tube secretory epithelium leads to high-grade serous ovarian cancer in Brca;Tp53;Pten models. Cancer Cell 24, 751-765 (2013).

4. Vigano, P., Somigliana, E., Chiodo, I., Abbiati, A. \& Vercellini, P. Molecular mechanisms and biological plausibility underlying the malignant transformation of endometriosis: a critical analysis. Hum. Reprod. Update 12, 77-89 (2006)

5. Ness, R. B. Endometriosis and ovarian cancer: thoughts on shared pathophysiology. Am. J. Obstet. Gynecol. 189, 280-294 (2003).

6. Kurman, R. J. \& Shih, I. e. M. The origin and pathogenesis of epithelial ovarian cancer: a proposed unifying theory. Am. J. Surg. Pathol. 34, 433-443 (2010).

7. Dinulescu, D. M. et al. Role of K-ras and Pten in the development of mouse models of endometriosis and endometrioid ovarian cancer. Nat. Med. 11, 63-70 (2005)

8. Wu, R. et al. Mouse model of human ovarian endometrioid adenocarcinoma based on somatic defects in the Wnt/beta-catenin and PI3K/Pten signaling pathways. Cancer Cell 11, 321-333 (2007).

9. Glasspool, R. M. \& McNeish, I. A. Clear cell carcinoma of ovary and uterus. Curr. Oncol. Rep. 15, 566-572 (2013).

10. Cho, K. R. \& Shih, I. e. M. Ovarian cancer. Annu. Rev. Pathol. 4, 287-313 (2009).

11. Tan, D. S., Miller, R. E. \& Kaye, S. B. New perspectives on molecular targeted therapy in ovarian clear cell carcinoma. Br. J. Cancer 108, 1553-1559 (2013).

12. Kobayashi, H. Ovarian cancer in endometriosis: epidemiology, natural history, and clinical diagnosis. Int. J. Clin. Oncol. 14, 378-382 (2009).

13. Jimbo, H. et al. Prevalence of ovarian endometriosis in epithelial ovarian cancer. Int. J. Gynaecol. Obstet. 59, 245-250 (1997).

14. Ness, R. B. Endometriosis and ovarian cancer: thoughts on shared pathophysiology. Am. J. Obstet. Gynecol. 189, 280-294 (2003).

15. Itamochi, H., Kigawa, J. \& Terakawa, N. Mechanisms of chemoresistance and poor prognosis in ovarian clear cell carcinoma. Cancer Sci. 99, 653-658 (2008)

16. Tan, D. S. \& Kaye, S. Ovarian clear cell adenocarcinoma: a continuing enigma. J. Clin. Pathol. 60, 355-360 (2007).

17. Jones, S. et al. Frequent mutations of chromatin remodeling gene ARID1A in ovarian clear cell carcinoma. Science 330, 228-231 (2010). 
18. Wiegand, K. C. et al. ARID1A mutations in endometriosis-associated ovarian carcinomas. New Engl. J. Med. 363, 1532-1543 (2010).

19. Wu, J. N. \& Roberts, C. W. ARID1A mutations in cancer: another epigenetic tumor suppressor? Cancer Discov. 3, 35-43 (2013).

20. Jones, S. et al. Genomic analyses of gynaecologic carcinosarcomas reveal frequent mutations in chromatin remodelling genes. Nat. Commun. 5, 5006 (2014).

21. Kadoch, C. et al. Proteomic and bioinformatic analysis of mammalian SWI/SNF complexes identifies extensive roles in human malignancy. Nat. Genet. 45, 592-601 (2013).

22. Wang, X., Haswell, J. R. \& Roberts, C. W. Molecular pathways: SWI/SNF (BAF) complexes are frequently mutated in cancer-mechanisms and potential therapeutic insights. Clin. Cancer Res. 20, 21-27 (2014).

23. Jones, S. et al. Somatic mutations in the chromatin remodeling gene ARID1A occur in several tumor types. Hum. Mutat. 33, 100-103 (2012).

24. Chandler, R. L. et al. ARIDla-DNA interactions are required for promoter occupancy by SWI/SNF. Mol. Cell. Biol. 33, 265-280 (2013).

25. Lengyel, E. et al. Epithelial ovarian cancer experimental models. Oncogene 33, 3619-3633 (2014).

26. Guan, B. et al. Roles of deletion of Arid1a, a tumor suppressor, in mouse ovarian tumorigenesis. J. Natl Cancer Inst. 106 pii: djul46 (2014).

27. Gao, X. et al. ES cell pluripotency and germ-layer formation require the SWI/SNF chromatin remodeling component BAF250a. Proc. Natl Acad. Sci. USA 105, 6656-6661 (2008).

28. Hayashi, S., Lewis, P., Pevny, L. \& McMahon, A. P. Efficient gene modulation in mouse epiblast using a Sox2Cre transgenic mouse strain. Mech. Dev. 119 (Suppl 1), S97-S101 (2002).

29. Flesken-Nikitin, A., Choi, K. C., Eng, J. P., Shmidt, E. N. \& Nikitin, A. Y. Induction of carcinogenesis by concurrent inactivation of $\mathrm{p} 53$ and $\mathrm{Rb} 1$ in the mouse ovarian surface epithelium. Cancer Res. 63, 3459-3463 (2003).

30. Campbell, I. G. et al. Mutation of the PIK3CA gene in ovarian and breast cancer. Cancer Res. 64, 7678-7681 (2004).

31. Kuo, K. T. et al. Frequent activating mutations of PIK3CA in ovarian clear cell carcinoma. Am. J. Pathol. 174, 1597-1601 (2009).

32. Zang, Z. J. et al. Exome sequencing of gastric adenocarcinoma identifies recurrent somatic mutations in cell adhesion and chromatin remodeling genes. Nat. Genet. 44, 570-574 (2012).

33. Yamamoto, S., Tsuda, H., Takano, M., Tamai, S. \& Matsubara, O. Loss of ARID1A protein expression occurs as an early event in ovarian clear-cell carcinoma development and frequently coexists with PIK3CA mutations. Mod. Pathol. 25, 615-624 (2012).

34. Zhao, L. \& Vogt, P. K. Helical domain and kinase domain mutations in p110alpha of phosphatidylinositol 3-kinase induce gain of function by different mechanisms. Proc. Natl Acad. Sci. USA 105, 2652-2657 (2008).

35. Adams, J. R. et al. Cooperation between Pik3ca and p53 mutations in mouse mammary tumor formation. Cancer Res. 71, 2706-2717 (2011).

36. Kinross, K. M. et al. An activating Pik3ca mutation coupled with Pten loss is sufficient to initiate ovarian tumorigenesis in mice. J. Clin. Invest. 122, 553-557 (2012).

37. Lowery, W. J. et al. Loss of ARID1A-associated protein expression is a frequent event in clear cell and endometrioid ovarian cancers. Int. J. Gynecol. Cancer 22, 9-14 (2012)

38. Offman, S. L. \& Longacre, T. A. Clear cell carcinoma of the female genital tract (not everything is as clear as it seems). Adv. Anat. Pathol. 19, 296-312 (2012).

39. Hendrix, N. D. et al. Fibroblast growth factor 9 has oncogenic activity and is a downstream target of Wnt signaling in ovarian endometrioid adenocarcinomas. Cancer Res. 66, 1354-1362 (2006).

40. Yamaguchi, K. et al. Identification of an ovarian clear cell carcinoma gene signature that reflects inherent disease biology and the carcinogenic processes. Oncogene 29, 1741-1752 (2010).

41. Yanaihara, N. et al. Cytokine gene expression signature in ovarian clear cell carcinoma. Int. J. Oncol. 41, 1094-1100 (2012).

42. Anglesio, M. S. et al. IL6-STAT3-HIF signaling and therapeutic response to the angiogenesis inhibitor sunitinib in ovarian clear cell cancer. Clin. Cancer Res. 17, 2538-2548 (2011).

43. Naugler, W. E. \& Karin, M. The wolf in sheep's clothing: the role of interleukin-6 in immunity, inflammation and cancer. Trends Mol. Med. 14, 109-119 (2008)

44. Guo, Y., Xu, F., Lu, T., Duan, Z. \& Zhang, Z. Interleukin-6 signaling pathway in targeted therapy for cancer. Cancer Treat. Rev. 38, 904-910 (2012).

45. Vasudevan, K. M. et al. AKT-independent signaling downstream of oncogenic PIK3CA mutations in human cancer. Cancer Cell 16, 21-32 (2009).

46. Jia, S. et al. Essential roles of PI(3)K-p110beta in cell growth, metabolism and tumorigenesis. Nature 454, 776-779 (2008).

47. Ness, R. B. \& Cottreau, C. Possible role of ovarian epithelial inflammation in ovarian cancer. J. Natl Cancer Inst. 91, 1459-1467 (1999).

48. Wiegand, K. C. et al. ARID1A mutations in endometriosis-associated ovarian carcinomas. New Engl. J. Med. 363, 1532-1543.
49. Samartzis, E. P. et al. Loss of ARID1A/BAF250a-expression in endometriosis: a biomarker for risk of carcinogenic transformation? Mod. Pathol. 25, 885-892 (2012).

50. Wiegand, K. C. et al. Loss of BAF250a (ARID1A) is frequent in high-grade endometrial carcinomas. J. Pathol. 224, 328-333.

51. Somigliana, E. et al. Use of the concomitant serum dosage of CA 125, CA 19-9 and interleukin- 6 to detect the presence of endometriosis. Results from a series of reproductive age women undergoing laparoscopic surgery for benign gynaecological conditions. Hum. Reprod. 19, 1871-1876 (2004).

52. Darai, E., Detchev, R., Hugol, D. \& Quang, N. T. Serum and cyst fluid levels of interleukin (IL) -6, IL-8 and tumour necrosis factor-alpha in women with endometriomas and benign and malignant cystic ovarian tumours. Hum. Reprod. 18, 1681-1685 (2003).

53. Chang, Q., Daly, L. \& Bromberg, J. The IL-6 feed-forward loop: a driver of tumorigenesis. Semin. Immunol. 26, 48-53 (2014).

54. Liu, P., Jenkins, N. A. \& Copeland, N. G. A highly efficient recombineeringbased method for generating conditional knockout mutations. Genome Res. 13, 476-484 (2003).

55. Brachmann, S. M. et al. Characterization of the mechanism of action of the pan class I PI3K inhibitor NVP-BKM120 across a broad range of concentrations. Mol. Cancer Ther. 11, 1747-1757 (2012).

56. Roberts, P. J. et al. Combined PI3K/mTOR and MEK inhibition provides broad antitumor activity in faithful murine cancer models. Clin. Cancer Res. 18, 5290-5303 (2012).

57. Chen, Z. et al. A murine lung cancer co-clinical trial identifies genetic modifiers of therapeutic response. Nature 483, 613-617 (2012).

58. Collaborative Cross Consortium. The genome architecture of the Collaborative Cross mouse genetic reference population. Genetics 190, 389-401 (2012).

59. Van Loo, P. et al. Allele-specific copy number analysis of tumors. Proc. Natl Acad. Sci. USA 107, 16910-16915 (2010).

60. Cerami, E. et al. The cBio cancer genomics portal: an open platform for exploring multidimensional cancer genomics data. Cancer Discov. 2, 401-404 (2012).

61. Gao, J. et al. Integrative analysis of complex cancer genomics and clinical profiles using the cBioPortal. Sci. Signal. 6, pl1 (2013).

62. Bolstad, B. M., Irizarry, R. A., Astrand, M. \& Speed, T. P. A comparison of normalization methods for high density oligonucleotide array data based on variance and bias. Bioinformatics 19, 185-193 (2003).

63. Smyth, G. K. Linear models and empirical bayes methods for assessing differential expression in microarray experiments. Stat. Appl. Genet. Mol. Biol. 3, Article3 (2004).

64. Tusher, V. G., Tibshirani, R. \& Chu, G. Significance analysis of microarrays applied to the ionizing radiation response. Proc. Natl Acad. Sci. USA 98, 5116-5121 (2001).

65. Subramanian, A. et al. Gene set enrichment analysis: a knowledge-based approach for interpreting genome-wide expression profiles. Proc. Natl Acad. Sci. USA 102, 15545-15550 (2005).

66. Mootha, V. K. et al. PGC-1alpha-responsive genes involved in oxidative phosphorylation are coordinately downregulated in human diabetes. Nat. Genet. 34, 267-273 (2003).

67. Shepherd, T. G., Theriault, B. L., Campbell, E. J. \& Nachtigal, M. W. Primary culture of ovarian surface epithelial cells and ascites-derived ovarian cancer cells from patients. Nat. Protoc. 1, 2643-2649 (2006).

68. Flesken-Nikitin, A. et al. Ovarian surface epithelium at the junction area contains a cancer-prone stem cell niche. Nature 495, 241-245 (2013).

69. Inouye, S. et al. Impaired IgG production in mice deficient for heat shock transcription factor 1. J. Biol. Chem. 279, 38701-38709 (2004).

70. Inouye, S. et al. Heat shock transcription factor 1 opens chromatin structure of interleukin-6 promoter to facilitate binding of an activator or a repressor. J. Biol. Chem. 282, 33210-33217 (2007).

\section{Acknowledgements}

We thank members of the Magnuson and Kim Lab and Drs Scott Bultman and Victoria Bae-Jump for helpful discussions. We thank Dr Anna Spagnoli for generously sharing imaging equipment, Jane Hoel and Jackie Brooks for help with the AdCRE injections, Mike Vernon for performing the microarray hybridizations and members of the Laboratory of Pardo-Manuel de Villena for technical assistance with the copy-number analysis. We also thank Drs Susan Murphy and Zhiqing Huang at Duke for their helpful discussions on primary ovarian tumour cell isolation methods. Dr Virginia Godfrey at UNC provided assistance with mouse tumour pathology. The UNC Lineberger Comprehensive Cancer Center's Mouse Phase One Unit (MP1U) provided assistance with the therapeutic studies. MP1U is supported by the University Cancer Research Fund. R.L.C. was supported by an American Cancer Society Postdoctoral Fellowship (PF-09-116-01-CCG) and an Ann Schreiber Mentored Investigator Award (258831) from the Ovarian Cancer Research Fund. J.S.D. was supported by the Integrative Vascular Biology Training Grant (T32-HL069768). W.Y.K. is a Damon Runyon Merck Clinical Investigator. This work was supported by an AACR Kurelt grant to W.Y.K. and NIH grants to W.Y.K. (CA142794) and T.M. (HD03665). 


\section{Author contributions}

R.L.C. conceived this study, developed the hypothesis and wrote the manuscript with supervision and gracious support from T.M. R.L.C., J.S.D., J.C.S., D.B.D., F.P.-M.d.V., W.Y.K. and T.M. contributed to the experimental design. R.L.C., J.S.D., J.C.S., D.S., D.Y. and J.X. performed the experiments. J.R.R. and J.P.D. performed the computational analysis with conceptual advice from M.D.W. and J.S. All the authors commented on the manuscript.

\section{Additional information}

Accession codes: Microarray gene expression data sets generated in this study were deposited in the Gene Expression Omnibus (GEO) repository under the accession code GSE57380.
Supplementary Information accompanies this paper at http://www.nature.com/ naturecommunications

Competing financial interests: The authors declare no competing financial interests.

Reprints and permission information is available online at http://npg.nature.com/ reprintsandpermissions/

How to cite this article: Chandler, R. L. et al. Coexistent ARID1A-PIK3CA mutations promote ovarian clear-cell tumorigenesis through pro-tumorigenic inflammatory cytokine signalling. Nat. Commun. 6:6118 doi: $10.1038 / \mathrm{ncomms7118}$ (2015). 